\title{
Assessment of spiritual suffering in the cancer context: A systematic literature review
}

\author{
MEGAN BEST, BMED(HONS), MA ${ }^{1}$ LYNLEY ALDRIDGE, BPSYCH, MA,${ }^{1}$ \\ PHYLLIS BUTOW, PHD, MPH, ${ }^{1}$ IAN OLVER, MD, PHD, ${ }^{2}$ MELANIE PRICE, PHD, ${ }^{1}$ AND \\ FLEUR WEBSTER, MSCVSC, $\mathrm{MPH}^{3}$ \\ ${ }^{1}$ Psycho-Oncology Co-operative Research Group (PoCoG), University of Sydney, Sydney, Australia \\ ${ }^{2}$ Cancer Council Australia, Sydney, Australia \\ ${ }^{3}$ Cancer Australia, Sydney, Australia \\ (ReCeived August 13, 2014; Accepted August 18, 2014)
}

\begin{abstract}
Objective: An important goal of cancer medicine is relief of patients' suffering. In view of the clinical challenges of identifying suffering patients, we sought to identify valid instruments for assessing the spiritual suffering of people diagnosed with cancer.

Method: A systematic review of the literature was conducted in the Medline, Embase, the Cochrane Library, and PsycINFO databases seeking assessment instruments that measure either suffering or one of its synonyms or symptoms. The psychometric properties of the identified measures were compared.

Results: A total of 90 articles were identified that supplied information about 58 measures. The constructs examined were: suffering, hopelessness/demoralization, hope, meaning, spiritual well-being, quality of life where a spiritual/existential dimension was included, distress in the palliative care setting and pain, distress or struggle of a spiritual nature. The Pictorial Representation of Illness and Self Measure (PRISM) (patient completed) was the most promising measure identified for measuring the burden of suffering caused by illness due to its ease of use and the inclusion of a subjective component.

Significance of Results: Although the appropriateness of any measure for the assessment of spiritual suffering in cancer patients will depend on the context in which it is intended to be utilized, the PRISM is promising for measuring the burden of suffering due to illness.
\end{abstract}

KEYWORDS: Cancer, Assessment, Suffering, Spirituality, Systematic review

\section{INTRODUCTION}

Optimal care of people with cancer incorporates the effective management of physical, psychological, social, and existential/spiritual well-being, and strives to alleviate suffering. Our recent systematic review investigating the experience and management of suffering in cancer (Best et al., 2014) concluded that spiritual suffering is defined as "an all-encompassing, dynamic, individual phenomenon characterized

Address correspondence and reprint requests to: Megan Best, PoCoG, Level 6 North, Lifehouse (C39Z), University of Sydney, New South Wales 2006, Australia. E-mail: megan.best@sydney. edu.au by the experience of alienation, helplessness, hopelessness, and meaninglessness in the sufferer that is difficult for them to articulate. It is multidimensional and usually incorporates an undesirable, negative quality." Surrogate terms, antecedents, and consequences of suffering were described and recommendations were made to address spiritual suffering in cancer patients. However, a reliable means for assessing suffering is needed in order to achieve this goal.

Potential barriers to recognition of suffering in cancer patients include the difficulty patients have in articulating their suffering, either due to an inability to find the appropriate vocabulary or an 
unwillingness to burden others (Boston et al., 2011; Cherny et al., 1994; Younger, 1995). Assistance may be needed to voice the conflict, which is known to be beneficial for the sufferer (Blinderman \& Cherny, 2005). However, healthcare workers may not be able to identify patient distress or may be unwilling to acknowledge it due to the biopsychosocial paradigm of Western medicine that ignores the spiritual (Arman et al., 2004; Ferrell, 1993). Healthcare staff may fail to respond to suffering even if they recognize it (Rodgers \& Cowles, 1997), perhaps because of their own death anxiety (Kahn \& Steeves, 1995). Patients may wait for a cue that never comes or just assume that the staff are too busy to listen (Strang, 1997). Some sufferers feel a lack of a "safe space" in which to discuss their fears (Moore et al., 2004). This situation highlights the need for reliable tools for assessment of suffering that are not dependent on patients finding the opportunity to voice their distress.

Information about the assessment of suffering in the context of cancer is not easily accessible, nor have the relative benefits and disadvantages of the available assessment tools been compared. (Rodin, 2003). Previous reviews of the assessment of suffering have been limited to the psychological aspects of distress (Carlson \& Bultz, 2003; Carlson et al., 2012; Kelly et al., 2006) or focused on the end-of-life setting (Krikorian et al., 2013). To address this gap in the literature, we undertook a systematic review of measures of spiritual suffering in people diagnosed with cancer, including currently treated, palliative, and survival populations.

\section{METHOD}

\section{Search}

Between April and June of 2012, a systematic search of the literature was conducted to identify all English-language studies published between 1992 and 2012 that focused on assessment of suffering in cancer patients. The following databases were systematically searched: MEDLINE, EMBASE, the Cochrane Library, and PSYCINFO. To ensure a sufficiently broad range of conceptualizations of suffering, the search strategy was drafted using an iterative process. Results from preliminary searches were employed to develop a list of concepts identified in the literature as synonymous with suffering, or potentially measurable "symptoms" of suffering and their antonyms (see Tables 1 and 2). The identified search terms (see Table 3) were entered in each of the databases listed above.

In order to be included, reports had to: (1) be published in a peer-reviewed journal; (2) focus on adults
Table 1. Terms used synonymously with "suffering"

Concept

Suffering

Existential distress

Existential suffering

Existential pain

Spiritual distress

Spiritual suffering

Spiritual pain

Psychospiritual distress

Psychoexistential suffering

Total pain

Demoralization

(aged 18 years and above) who had been diagnosed with cancer; (3) report on outcomes relevant to the review question (i.e., assessment of suffering in cancer patients); and (4) assess tools/instruments that measured either suffering or one of its synonyms or symptoms (as listed in Tables 1 and 2).

Reports were excluded if they: (1) focused on children with cancer, parents of children with cancer, other carers of patients with cancer, or adult survivors of childhood cancers; (2) focused on suffering in patient groups with and without cancer, unless the results were reported separately for cancer patients, or unless the sample was predominantly cancer patients (e.g. 95\% or more); (3) were books, book chapters, dissertation abstracts, or conference abstracts; (4) utilized or reviewed measures of interest without reporting the psychometric properties of the instrument; or (5) focused predominantly on spiritual or existential "issues" or "concerns." Articles fitting within this last category were closely reviewed to determine whether they simply explored spiritual or existential aspects of life that might be impacted (positively or negatively) by a cancer diagnosis and might or might not lead to suffering, or whether

Table 2. Potentially measurable "symptoms" of suffering (and their "opposites")

\author{
Concept \\ Hopelessness, despair \\ Loss of meaning \\ Sense of meaning/finding meaning \\ Sense of coherence \\ Purpose in life \\ Hope \\ Dignity \\ Transcendence \\ Spiritual well-being \\ Peace \\ Faith \\ Crisis of faith \\ Desire for (hastened) death
}


Table 3. Search terms

\begin{tabular}{ll}
\hline \hline Search Terms & \\
Suffering & Hope \\
Existentialism & Hopelessness \\
Meaning & Faith \\
Purpose & Peace \\
Transcendence & Sense of coherence \\
Spirituality & Demoralization \\
Hope & Dignity \\
Total pain & Neoplasm \\
Cancer & \\
& \\
\hline \hline
\end{tabular}

they were in fact reporting on "distress," "pain," "crisis," "anguish," or another synonym of "suffering." There are a number of existing review papers that explore the former group of papers (Bresnahan \& Merrill, 2000; Henoch \& Danielson, 2009; Sulmasy, 2006). For these reasons, only the second group of papers were included in the present review.

All retrieved articles were reviewed against the selection criteria, and manual searches were conducted to identify any additional relevant articles not retrieved by the systematic search. Articles that employed or reviewed measures of interest without reporting the psychometric properties of the instrument (and therefore excluded from the search) were separately reviewed to generate a list of additional measures for which instrument development/validation studies were subsequently sought. We attempted as far as possible to include initial and key publications pertaining to the psychometric properties of an instrument, particularly if it was reported in the cancer context. Papers summarizing psychometric properties for a measure across multiple studies were deemed eligible for inclusion if no specific studies in the cancer context were available.

A flowchart presenting the results of the literature search is presented as Figure 1.

\section{Data Extraction}

For each instrument/validation study the following data were extracted by LA and MB:

1. Properties of the measure: mode of administration; number of items; response scale; scoring.

2. Details of the initial and key validation samples.

3. Details of the item development process.

4. Information on any domains/subscales.

5. Information on reliability, validity, and responsiveness to change.

\section{Criteria for Evaluating Outcome}

Individual assessment tools (not studies) were evaluated by LA and MB according to Fitzpatrick et al.'s (1998) criteria-namely, appropriateness (is the content of the instrument appropriate to the questions asked?), reliability (does the instrument produce results that are reproducible and internally consistent?), validity (does the instrument measure what it claims to measure?), responsiveness (does the instrument detect changes over time that matter to patients?), precision (how precise are the scores of the instrument?), interpretability (how interpretable are the scores of the instrument?), and acceptability (is the instrument acceptable to patients?). Data pertaining to the psychometric properties of individual measures were extracted, as this was deemed the best way of presenting evidence about the appropriateness of each outcome measure. Evaluations were completed and reviewed individually first, then discussed as a group until consensus was reached. Psychometric properties were rated according to whether the bulk of the available evidence was supportive (+), not supportive (-), or whether assessment of the property had either given contradictory results or not been assessed (?).

\section{RESULTS}

Systematic searches of the literature resulted in identification of 90 articles presenting information about 58 measures, which appeared to assess either suffering or one of its synonyms or symptoms. The constructs examined by the eligible measures were: suffering, hopelessness/demoralization, hope, meaning, spiritual well-being, quality of life where a spiritual/existential dimension was included, distress in the palliative care setting and pain, distress or struggle of a spiritual nature. The psychometric properties of the selected measures are set forth in Table 4.

\section{Suffering}

Two measures of suffering for which psychometric properties are available were identified: the MiniSuffering State Examination (MSSE) (physician completed) (Aminoff et al., 2004) and the Pictorial Representation of Illness and Self Measure (PRISM) (patient completed) (Büchi et al., 2002).

\section{Mini-Suffering State Examination (MSSE)}

The MSSE is a brief clinician-administered measure of suffering that may be particularly useful with endstage cancer patients who experience difficulties communicating their needs and/or expressing their 


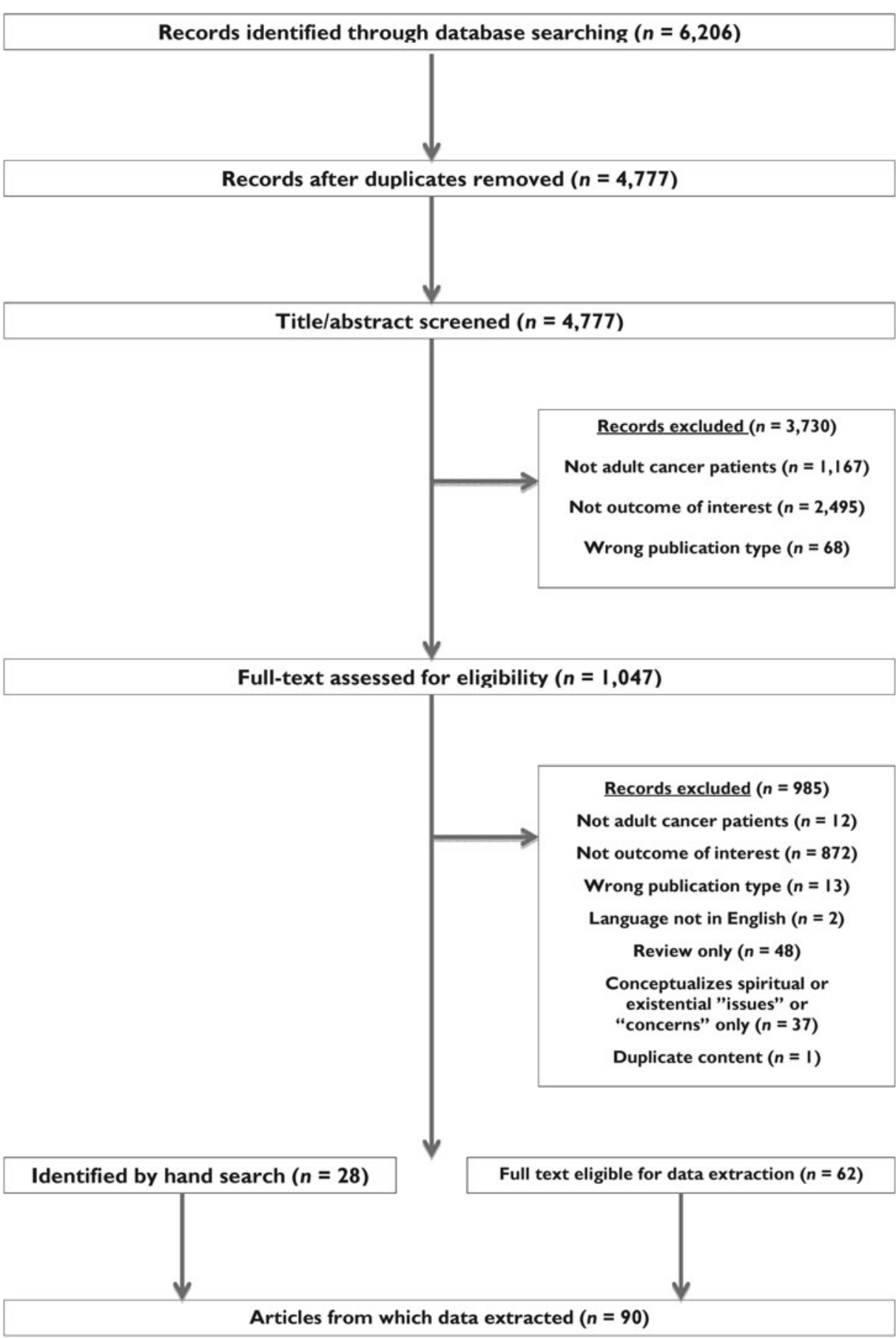

Fig. 1. Flowchart of literature search.

suffering (Adunsky et al., 2008). It was originally developed in the context of dementia (Aminoff et al., 2004), but preliminary work has been done to explore its psychometric properties in the context of cancer (Adunsky et al., 2008).

Content validity is dependent on the clinical judgment of the scale's designers, and there is no indication of further work seeking confirmation of appropriateness and comprehensiveness of items. The 10 items included on this scale do not necessarily encompass the full range of, nor even the most pressing dimensions of, suffering in cancer patients at the end of life, suggesting that this tool may be a useful starting point for measuring some types of suffering, but that further work exploring its content validity may be required.

Reliability overall appears adequate, though some of the items are fairly subjective (e.g., "suffering according to medical opinion" and "not calm"), and this was reflected by lower levels of observer agreement ( $\kappa=0.62$ and 0.64$)$ on ratings for these two items (Aminoff et al., 2004). 
Table 4. Psychometric properties of identified assessment tools

\begin{tabular}{|c|c|c|c|c|c|c|c|}
\hline Construct Measured & Tool & Description & Cancer Validation* & Domains & Reliability & $\begin{array}{l}\text { Construct Validity } \\
(+/ ? /-)^{* * *}\end{array}$ & $\begin{array}{l}\text { Responsiveness to } \\
\text { Change }(+/ ? /-)^{* * *}\end{array}$ \\
\hline Suffering & $\begin{array}{l}\text { MSSE (Aminoff et al., } \\
\text { 2004) }\end{array}$ & $\begin{array}{l}\text { Administration: } \\
\text { clinician-rated } \\
\text { Items: } 10 \\
\text { Response scale: } \\
\text { yes/no }(1 / 0) \\
\text { Scores: } \\
\text { range } 0-10 \\
0-3 \text { reflects low } \\
\text { level of suffering, } \\
4-6 \text { intermediate, } \\
7-10 \text { high }\end{array}$ & $\begin{array}{l}152 \text { advanced cancer } \\
\text { patients with }<1 \\
\text {-month life } \\
\text { expectancy } \\
\text { Israel (Adunsky } \\
\text { et al., 2008) }\end{array}$ & None specified & $\begin{array}{l}\text { Physician 1, internal } \\
\text { consistency: } \\
\alpha=0.735 \text { (Aminoff et al., } \\
2004 \text { ) } \\
\text { Physician 2, internal } \\
\text { consistency: } \\
\alpha=0.718 \text { (Aminoff et al., } \\
2004 \text { ) } \\
\text { Interobserver reliability: } \\
\kappa=0.791 \text { (Aminoff et al., } \\
2004 \text { ) } \\
\text { Internal consistency: } \\
\alpha=0.738 \text { (Adunsky } \\
\text { et al., 2008) }\end{array}$ & $\begin{array}{l}+ \text { (Aminoff et al., } \\
\text { 2004) } \\
\text { ? (Adunsky et al., } \\
\text { 2008) }\end{array}$ & $+\underset{2008)}{\text { (Adunsky et al., }}$ \\
\hline " & $\begin{array}{l}\text { PRISM (Büchi et al., } \\
\text { 2002) }\end{array}$ & $\begin{array}{l}\text { Administration: } \\
\text { clinician } \\
\text { administered and/ } \\
\text { or self-report (by } \\
\text { post with stickers) } \\
\text { Items: } 1 \\
\text { Response scale: } \\
\text { placement of } \\
\text { "illness" disc on } \\
\text { board. Perceived } \\
\text { burden of suffering } \\
\text { due to illness is } \\
\text { distance in cm from } \\
\text { centre of "illness" } \\
\text { disc to center of } \\
\text { "self" disc } \\
\text { Size of "illness" disc } \\
\text { (PRISM-R2) } \\
\text { represents } \\
\text { perceived severity } \\
\text { of illness, range } \\
\text { 1-3 (for three sizes } \\
\text { of disc) }\end{array}$ & $\begin{array}{l}1299 \text { long-term } \\
\text { survivors of } \\
\text { prostate cancer, } \\
\text { endometrial } \\
\text { cancer, non- } \\
\text { Hodgkin's and } \\
\text { Hodgkin's } \\
\text { lymphoma } \\
\text { The Netherlands } \\
\text { (Wouters et al., } \\
\text { 2011) }\end{array}$ & None specified & $\begin{array}{l}\text { Test-retest reliability ( } 2 \\
\text { hours) } r=0.95 \text { (Büchi } \\
\text { et al., 2002) } \\
\text { Interrater reliability } \\
(6 \text { hours) } \mathrm{r}=0.79 \text { (Büchi } \\
\text { et al., 2002) }\end{array}$ & $\begin{array}{l}\text { + (Büchi et al., 2002; } \\
\text { Büchi \& Sensky, } \\
\text { 1999; Wouters } \\
\text { et al., 2011; } \\
\text { Wouters et al., } \\
\text { 2008a) }\end{array}$ & $\begin{array}{l}\text { + (Büchi et al., 2002; } \\
\text { Wouters et al., } \\
\text { 2008a) }\end{array}$ \\
\hline $\begin{array}{l}\text { Hopelessness/ } \\
\text { demoralization }\end{array}$ & $\begin{array}{l}\text { BHS (Beck et al., } \\
\text { 1974) }\end{array}$ & $\begin{array}{l}\text { Administration: } \\
\text { self-report } \\
\text { Items: } 20 \\
\text { Response scale: } \\
\text { true/false }(0 / 1) \\
\text { Scores: } \\
\text { range } 0-20 \text {. } \\
\text { Score of } 8 \text { or more } \\
\text { reflects moderate to } \\
\text { severe levels of } \\
\text { hopelessness (Beck } \\
\text { et al., 1993) }\end{array}$ & $\begin{array}{l}200 \text { hospice } \\
\text { inpatients with a } \\
\text { diagnosis of cancer } \\
\text { and a life expec- } \\
\text { tancy of less than } 6 \\
\text { months. } \\
\text { United States } \\
\text { (Abbey et al., 2006) } \\
406 \text { ambulatory } \\
\text { patients with } \\
\text { advanced lung or } \\
\text { gastrointestinal } \\
\text { cancer } \\
\text { Canada (Nissim } \\
\text { et al., 2010) }\end{array}$ & $\begin{array}{l}\text { Three factors identified in } \\
\text { initial study: feelings } \\
\text { about the future }(5 \text { items); } \\
\text { loss of motivation ( } 8 \\
\text { items); future } \\
\text { expectations ( } 5 \text { items) } 161 \\
\text { Two factors identified in } \\
\text { one cancer validation } \\
\text { study: } \\
\text { negative expectations ( } 10 \\
\text { items; } \alpha=0.86) ; \text { loss of } \\
\text { motivation ( } 10 \text { items; } \\
\alpha=0.83)\end{array}$ & $\begin{array}{l}\text { Internal consistency: } \\
\alpha=0.93 \text { (Beck et al., } \\
1974 \text { ) } \\
\text { Internal consistency } \\
20 \text {-item: } \\
\alpha=0.87 \\
\text { 3-item: } \\
\alpha=0.69 \text { (Abbey et al., } \\
2006 \text { ) } \\
7 \text {-item: } \\
\alpha=0.85 \text { (Abbey et al., } \\
2006 \text { ) } \\
13 \text {-item: } \alpha=0.89 \text { (Abbey } \\
\text { et al., } 2006 \text { ) }\end{array}$ & $\begin{array}{c}+ \text { (Abbey et al., 2006; } \\
\text { Beck et al., 1974; } \\
\text { Nissim et al., 2010) }\end{array}$ & $\begin{array}{l}\text { ? (Abbey et al., 2006; } \\
\text { Beck et al., 1974; } \\
\text { Nissim et al., 2010) } \\
\text { + (Northouse } \\
\text { et al., 2007) }\end{array}$ \\
\hline
\end{tabular}




\begin{tabular}{|c|c|c|c|c|c|c|c|}
\hline Construct Measured & Tool & Description & Cancer Validation* & Domains & Reliability & $\begin{array}{l}\text { Construct Validity } \\
(+/ ? /-)^{* *}\end{array}$ & $\begin{array}{l}\text { Responsiveness to } \\
\text { Change }(+/ ? /-)^{* * *}\end{array}$ \\
\hline “ & $\begin{array}{l}\text { CCM (Fortner et al., } \\
2003 \text { ) }\end{array}$ & $\begin{array}{l}\text { Administration: } \\
\text { self-report (paper } \\
\text { or tablet) } \\
\text { Items: } 7 \\
\text { Response scale: } \\
\text { 10-point Likert- } \\
\text { type scales }(0=\text { not } \\
\text { a problem; } 10=\text { as } \\
\text { bad as possible) } \\
\text { Scores: raw scores } \\
\text { are converted to } \\
\text { normalized t scores } \\
\text { with mean of } 50 \\
\text { and SD of } 10\end{array}$ & $\begin{array}{l}\text { Three adult cancer } \\
\text { outpatient } \\
\text { samples } \\
(n=449) \\
\text { United States } \\
\text { (Fortner et al., } \\
\text { 2003) }\end{array}$ & None specified & $\begin{array}{l}\text { Internal consistency (for all } \\
3 \text { samples combined) } \\
\alpha=0.89 \\
\text { Alternate forms pen and } \\
\text { paper and tablet: } \\
r=0.87\end{array}$ & + & $\begin{array}{l}\text { ?(Fortner et al., 2003) } \\
\quad+(\text { Abernethy } \\
\text { et al., 2010) }\end{array}$ \\
\hline “ & $\begin{array}{l}\text { Demoralization scale } \\
\text { (Jacobsen et al., } \\
2006 \text { ) }\end{array}$ & $\begin{array}{l}\text { Administration: } \\
\text { self-report } \\
\text { Items: } 7 \\
\text { Response options } \\
\text { and scoring not } \\
\text { specified }\end{array}$ & $\begin{array}{l}242 \text { advanced cancer } \\
\text { patients } \\
\text { United States } \\
\text { (Jacobsen et al., } \\
\text { 2006) }\end{array}$ & None specified & $\begin{array}{l}\text { Internal consistency: } \\
\quad \alpha=0.78\end{array}$ & + & $?$ \\
\hline " & $\begin{array}{l}\text { Demoralization scale } \\
\text { (Kissane et al., } \\
2004 \text { ) }\end{array}$ & $\begin{array}{l}\text { Administration: } \\
\text { self-report } \\
\text { Items: } 24 \\
\text { Response scale: } \\
\text { 5-point }(0=\text { never, } \\
1=\text { seldom, } \\
2=\text { sometimes, } \\
3=\text { often, } 4=\text { all } \\
\text { the time) } \\
\text { Scores: } \\
\text { range } 0-96\end{array}$ & $\begin{array}{l}100 \text { patients with } \\
\text { advanced cancer. } \\
\text { Australia (Kissane } \\
\text { et al., 2004) } \\
100 \text { inpatients } \\
\text { with advanced } \\
\text { cancer. } \\
\text { Ireland (Mullane } \\
\text { et al., 2009) }\end{array}$ & $\begin{array}{l}\text { Loss of meaning } \\
\quad \text { (5 items) } \\
\alpha=0.87 \\
\text { Dysphoria } \\
\text { (5 items) } \\
\alpha=0.85 \\
\text { Disheartenment } \\
\text { (6 items) } \\
\alpha=0.89 \\
\text { Helplessness } \\
\text { (4 items) } \\
\alpha=0.84 \\
\text { Sense of failure } \\
\text { (4 items) } \\
\alpha=0.71\end{array}$ & $\begin{array}{l}\text { Internal consistency: } \\
\quad \alpha=0.94\end{array}$ & $\begin{array}{l}+ \text { (Kissane et al., } \\
2004 \text { ) } \\
\quad \text { (Mullane et al., } \\
\quad 2009 \text { ) }\end{array}$ & $?$ \\
\hline “ & $\begin{array}{l}\text { HAI (Rosenfeld et al., } \\
\text { 2011) }\end{array}$ & $\begin{array}{l}\text { Administration: self- } \\
\text { report } \\
\text { Items: } 8 \\
\text { Response scale: } \\
\text { anchored } \\
\text { statements each } \\
\text { with three response } \\
\text { options }(0-2) \\
\text { Scores: } \\
\text { range } 0-16\end{array}$ & $\begin{array}{l}\text { Two advanced cancer } \\
\text { samples } \\
\quad(n=583)\end{array}$ & $\begin{array}{l}\text { Factor analysis supported a } \\
\text { one-factor model }\end{array}$ & $\begin{array}{l}\text { Internal consistency: } \\
\quad \alpha=0.87 \\
\text { Average interitem } \\
\text { correlation } \mathrm{r}=0.48\end{array}$ & + & $?$ \\
\hline
\end{tabular}




\begin{tabular}{|c|c|c|c|c|c|c|c|}
\hline " & $\begin{array}{l}\text { SISC (Wilson et al., } \\
2004 \text { ) }\end{array}$ & $\begin{array}{l}\text { Administration: } \\
\text { clinician- } \\
\text { administered } \\
\text { Items: } 1 \\
\text { Response scale: } \\
7 \text {-point scale } \\
\text { (0= none, } \\
1=\text { minimal, } \\
2=\text { mild, } \\
3=\text { moderate, } \\
4=\text { strong, } \\
5=\text { severe, } \\
6=\text { extreme) } \\
1 \text { or } 2 \text { indicates the } \\
\text { experience of the } \\
\text { symptom or } \\
\text { concern is } \\
\text { relatively low. } \\
3 \text { corresponds to an } \\
\text { issue that is } \\
\text { generally a } \\
\text { significant } \\
\text { problem. Higher } \\
\text { scores are } \\
\text { associated with } \\
\text { clear presence of a } \\
\text { symptom or } \\
\text { concern at a } \\
\text { clinically } \\
\text { important level } \\
\text { with varying } \\
\text { degrees of severity. }\end{array}$ & $\begin{array}{l}69 \text { palliative care } \\
\text { (advanced) cancer } \\
\text { patients } \\
\text { Canada }\end{array}$ & Single item & $\begin{array}{l}\text { Interrater reliability } \\
\quad r=0.98 \\
\text { Test-retest } \\
(1-3 \text { days }) \\
r=0.80\end{array}$ & + & ? \\
\hline Hope & $\begin{array}{l}\text { ADHS (Snyder et al., } \\
1991 \text { ) }\end{array}$ & $\begin{array}{l}\text { Administration: self- } \\
\text { report } \\
\text { Items: } \\
8 \text { hope items }+4 \\
\text { filler items } \\
\text { Response scale: } \\
\text { 4-point scale (1= } \\
\text { definitely false, } \\
2=\text { mostly false, } \\
3=\text { mostly true, } \\
4=\text { definitely true) } \\
\text { Scores: } \\
\text { range } 12-48 \\
\text { Higher score } \\
\text { indicates higher } \\
\text { hope }\end{array}$ & & $\begin{array}{l}\text { Agency: } \\
\quad \begin{array}{l}\alpha=0.71-0.76 \\
\text { Pathways: } \\
\alpha=0.63-0.80\end{array}\end{array}$ & $\begin{array}{l}\text { Internal consistency: } \\
\quad \alpha=0.74-0.84 \\
\text { Test-retest reliability } \\
\text { (3-week) } r=0.85 \\
\text { (8-week) } r=0.73 \\
\text { (10-week } \\
r=0.76 \\
r=0.82\end{array}$ & + & + (Fallah et al., 2011) \\
\hline
\end{tabular}




\begin{tabular}{|c|c|c|c|c|c|c|c|}
\hline Construct Measured & Tool & Description & Cancer Validation* & Domains & Reliability & $\begin{array}{l}\text { Construct Validity } \\
(+/ ? /-)^{* * *}\end{array}$ & $\begin{array}{l}\text { Responsiveness to } \\
\text { Change }(+/ ? /-)^{* *}\end{array}$ \\
\hline " & HHS (Herth, 1991) & $\begin{array}{l}\text { Administration: self- } \\
\text { report } \\
\text { Items: } 30 \\
\text { Response scale: } \\
\text { 4-point rating scale } \\
\text { (0-3, where 0 } \\
\text { indicates that the } \\
\text { statement never } \\
\text { applies) } \\
\text { Scores: } \\
\text { range } 0-90 \\
\text { higher scores } \\
\text { denote greater } \\
\text { hope }\end{array}$ & $\begin{array}{l}180 \text { cancer patients } \\
\text { (NB: this group } \\
\text { was used for } \\
\text { pretesting and } \\
\text { pilot testing-not } \\
\text { the final } \\
\text { validation) United } \\
\text { States }\end{array}$ & $\begin{array}{l}\text { Temporality and future } \\
\text { (cognitive-temporal) } \\
\alpha=0.91 \\
\text { Positive readiness and } \\
\text { expectancy (affective- } \\
\text { behavioral) } \\
\alpha=0.90 \\
\text { Interconnectedness } \\
\text { (affiliative-contextual) } \\
\alpha=0.87\end{array}$ & $\begin{array}{l}\text { Internal consistency } \\
\quad \text { (population): } \\
\text { Well adults } \\
\alpha=0.92 \\
\text { Well elderly } \\
\alpha=0.94 \\
\text { Elderly widow(er)s } \\
\alpha=0.95 \\
\text { Test-retest reliability } \\
\text { (3-week): } \\
\text { Well adults } \\
r=0.90 \\
\text { Well elderly } \\
r=0.89 \\
\text { Elderly widow }(\mathrm{er}) \mathrm{s} \\
r=0.91\end{array}$ & 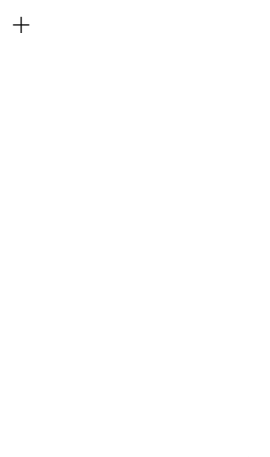 & $?$ \\
\hline “ & HHI (Herth, 1992) & $\begin{array}{l}\text { Administration: self- } \\
\text { report } \\
\text { Items: } 12 \\
\text { Response scale: } \\
1 \text { (strongly } \\
\text { disagree) to } 4 \\
\text { (strongly agree) } \\
\text { Scores: } \\
\text { range } 12-48 \\
\text { Higher scores } \\
\text { indicate a higher } \\
\text { level of hope }\end{array}$ & $\begin{array}{l}\text { Two groups of adoles- } \\
\text { cents and young } \\
\text { adults with cancer } \\
\text { (127 at various } \\
\text { stages of } \\
\text { treatment and } 74 \\
\text { newly diagnosed) } \\
\text { United States, } \\
\text { Canada (Phillips- } \\
\text { Salimi et al., 2007) }\end{array}$ & $\begin{array}{l}\text { Temporality and future } \\
\text { (cognitive-temporal) } \\
\text { Positive readiness and } \\
\text { expectancy (affective- } \\
\text { behavioral) } \\
\text { Interconnectedness } \\
\text { (affiliative-contextual) }\end{array}$ & $\begin{array}{l}\text { Internal consistency: } \\
\text { Acute } \alpha=0.98 \\
\text { Chronic } \alpha=0.96 \\
\text { Terminal } \alpha=0.94 \\
\text { Entire sample } \alpha=0.97 \\
\text { Domains: } \\
\alpha=0.78-0.86 \text { (Herth, } \\
\text { 1992) } \\
\text { Test-retest reliability } \\
(2 \text {-week) } \\
r=0.91 \text { (Herth, 1992) } \\
\text { Internal consistency: } \\
\text { Various stages } \\
\alpha=0.84 \\
\text { Newly diagnosed } \\
=0.78 \\
\text { (Herth 1992; Phillips- } \\
\text { Salimi et al., 2007) }\end{array}$ & + (Herth et al., 2007) & $+($ Herth, 2000) \\
\hline “ & $\begin{array}{l}\text { HD (Nekolaichuk } \\
\quad \text { et al., 1999) }\end{array}$ & $\begin{array}{l}\text { Administration: self- } \\
\text { report } \\
\text { Items: } 24 \\
\text { Response scale: } \\
\text { 7-point } \\
\text { (1= extremely, } \\
2=\text { quite, } \\
3=\text { slightly, } \\
4=\text { both or neither, } \\
5=\text { slightly, } 6 \text { = } \\
\text { quite, } \\
7=\text { extremely - } \\
\text { where } 1 \text { was the } \\
\text { negative response } \\
\text { and } 7 \text { the positive) } \\
\text { Scores: } \\
\text { range } 7-168 \\
\text { Higher scores } \\
\text { indicate an } \\
\text { enhanced hope } \\
\text { experience }\end{array}$ & & $\begin{array}{l}\text { Personal spirit (personal } \\
\text { dimensions) } \\
\text { Risk (situational } \\
\text { dimensions) } \\
\text { Authentic caring } \\
\text { (interpersonal dimension) }\end{array}$ & Not specified & + & $?$ \\
\hline
\end{tabular}




\begin{tabular}{|c|c|c|c|c|c|c|c|}
\hline " & $\begin{array}{l}\text { HDS (Nekolaichuk \& } \\
\text { Bruera, 2004) }\end{array}$ & $\begin{array}{l}\text { Administration: self- } \\
\text { report } \\
\text { Items: } 9 \\
\text { Response scale: } 1 \text { to } \\
7 \text { (1= extremely, } \\
2=\text { quite, } \\
3=\text { slightly, } \\
4=\text { both or neither, } \\
5=\text { slightly, } \\
6=\text { quite, } \\
7=\text { extremely, } \\
\text { where } 1 \text { was the } \\
\text { negative response } \\
\text { and } 7 \text { the positive) } \\
\text { Scores: } \\
\text { range } 7-63 \\
\text { Higher scores } \\
\text { indicate an } \\
\text { enhanced hope } \\
\text { experience }\end{array}$ & $\begin{array}{l}96 \text { advanced } \\
\text { (palliative) cancer } \\
\text { patients } \\
\text { Canada }\end{array}$ & $\begin{array}{c}\text { Authentic spirit } \\
\alpha=0.83 \\
\text { Comfort } \\
\alpha=0.69\end{array}$ & $\begin{array}{l}\text { Internal consistency: } \\
\quad \alpha=0.83\end{array}$ & + & $?$ \\
\hline “ & $\begin{array}{l}\text { MHS (Miller \& } \\
\quad \text { Powers, 1988) }\end{array}$ & $\begin{array}{l}\text { Administration: self- } \\
\text { report } \\
\text { Items: } 40 \\
\text { Response scale: } \\
\text { 5-point Likert-type } \\
\text { format from 5 } \\
\text { (strongly agree) to } \\
1 \text { (strongly } \\
\text { disagree) } \\
\text { Scores: } \\
\text { range } 40-200 \\
\text { Higher score } \\
\text { indicates high hope }\end{array}$ & & $\begin{array}{l}\text { Satisfaction with self, others } \\
\text { and life } \\
\text { Avoidance of hope threats } \\
\text { Anticipation of a future }\end{array}$ & 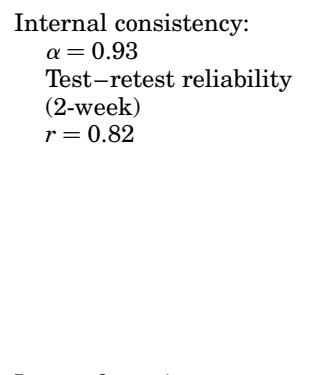 & + & 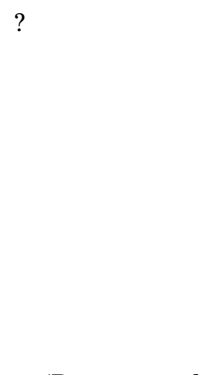 \\
\hline " & NHS (Nowotny, 1989) & $\begin{array}{l}\text { Administration: self- } \\
\text { report } \\
\text { Items: } 29 \\
\text { Response scale: } \\
\text { 4-point Likert-type } \\
\text { (strongly agree, } \\
\text { agree, disagree, } \\
\text { and strongly agree) } \\
\text { Scores: } \\
\text { range } 29-119 \\
\text { Higher scores } \\
\text { indicate high hope }\end{array}$ & $\begin{array}{l}306 \text { adults, (150 with } \\
\text { and } 156 \text { without } \\
\text { cancer), who had } \\
\text { experienced a } \\
\text { stressful event. } \\
\text { United States }\end{array}$ & $\begin{array}{l}\text { Confidence: } \\
\text { relates to others } \\
\text { Future is possible } \\
\text { Spiritual beliefs } \\
\text { Active involvement } \\
\text { Comes from within }\end{array}$ & $\begin{array}{l}\text { Internal consistency: } \\
\text { Overall: } \\
\alpha=0.90 \\
\text { Subscales: } \\
\alpha=0.6 \text { to } 0.9\end{array}$ & + & $+\underset{1998)}{(\text { Rustoen et al., }}$ \\
\hline Meaning & $\begin{array}{l}\text { CCCS (Chan et al., } \\
\text { 2007) }\end{array}$ & $\begin{array}{l}\text { Administration: self- } \\
\text { report } \\
\text { Items: } 11 \\
\text { (from original 24) } \\
\text { Response options } \\
\text { and scoring not } \\
\text { specified }\end{array}$ & $\begin{array}{l}190 \text { women with } \\
\text { breast cancer } \\
\text { (newly diagnosed } \\
\text { and survivors) (a) } \\
200 \text { women with } \\
\text { breast cancer } \\
\text { (within 2 years of } \\
\text { diagnosis without } \\
\text { metastasis) (b) } \\
\text { Hong Kong }\end{array}$ & $\begin{array}{l}\text { Incoherent-embittered } \\
\quad(6 \text { items }) \\
\alpha=0.86 \\
\text { (a) Coherent-enlightened } \\
\text { (5 items) } \\
\alpha=0.89 \\
\text { Confirmatory factor } \\
\text { analysis } \\
\text { (b) }\end{array}$ & $\begin{array}{l}\text { Test-retest reliability } \\
\quad(n=17 ; 4 \text { months): } \\
\text { Coherent-enlightened: } \\
r=0.87 \\
\text { Incoherent-embittered: } \\
r=0.89\end{array}$ & + & ? \\
\hline
\end{tabular}

Continued 


\begin{tabular}{|c|c|c|c|c|c|c|c|}
\hline Construct Measured & Tool & Description & Cancer Validation* & Domains & Reliability & $\begin{array}{l}\text { Construct Validity } \\
(+/ ? /-)^{* *}\end{array}$ & $\begin{array}{l}\text { Responsiveness to } \\
\text { Change }(+/ ? /-)^{* * *}\end{array}$ \\
\hline “ & $\begin{array}{l}\text { Constructed meaning } \\
\text { scale (Fife, 1995) }\end{array}$ & $\begin{array}{l}\text { Administration: self- } \\
\text { report } \\
\text { Items: } 8 \\
\text { Response scale: } \\
\text { 4-point (1-4: } \\
\text { strongly disagree; } \\
\text { disagree; agree; } \\
\text { strongly agree) } \\
\text { Scores: } \\
\text { range } 8-32\end{array}$ & $\begin{array}{l}422 \text { persons with a } \\
\text { variety of types of } \\
\text { cancer, at specified } \\
\text { points in the } \\
\text { illness trajectory. } \\
\text { United States }\end{array}$ & None specified & $\begin{array}{l}\text { Internal consistency } \\
\quad \alpha=0.81\end{array}$ & + & $?$ \\
\hline “ & $\begin{array}{l}\text { FACIT-Sp meaning } \\
\text { subscale (Canada } \\
\text { et al., 2008) }\end{array}$ & $\begin{array}{l}\text { Administration: self- } \\
\text { report } \\
\text { Items: } 12 \\
\text { Response scale: } \\
5 \text {-point }(0=\text { not at } \\
\text { all, } 1=\text { a little bit, } \\
2=\text { somewhat, } \\
3=\text { quite a bit, } \\
4=\text { very much }) \\
\text { Scores: } \\
\text { range } 0-48\end{array}$ & $\begin{array}{l}\text { 1,617 respondents, } \\
\text { predominant-ly } \\
\text { with cancer } \\
\text { diagnoses (a) } \\
131 \text { cancer } \\
\text { patients with } \\
\text { mixed early stage } \\
\text { and metastatic } \\
\text { diagnoses } \\
\text { (b) } \\
240 \text { long-term } \\
\text { female cancer } \\
\text { survivors } \\
\text { (c) } \\
\text { 8805 cancer } \\
\text { survivors (d). } \\
\text { United States, } \\
\text { Puerto Rico }\end{array}$ & $\begin{array}{l}\text { Meaning/faith subscale } \\
\text { (8 items) } \\
\alpha=0.81 \text { (a) } \\
\alpha=0.81 \text { (b) } \\
\text { Range: } 0-32 \\
\text { Meaning subscale } \\
\text { (4 items) } \\
\alpha=0.78 \text { (c) } \\
\alpha=0.78 \text { (d) } \\
\text { Range: } 0-16 \\
\text { Peace subscale } \\
\text { (4 items) } \\
\alpha=0.83 \text { (c) } \\
\alpha=0.83 \text { (d) } \\
\text { Range: } 0-16 \\
\text { Faith } \\
(4 \text { items) } \\
\alpha=0.88 \text { (a) } \\
\alpha=0.86 \text { (b) } \\
\alpha=0.84 \text { (c) } \\
\alpha=0.87 \text { (d) } \\
\text { Range: } 0-16\end{array}$ & $\begin{array}{l}\text { Internal consistency: } \\
\qquad \begin{array}{l}\alpha=0.87 \\
\alpha=0.86\end{array}\end{array}$ & $=/ ?$ & $\begin{array}{l}\text { Evidence of } \\
\text { responsiveness to } \\
\text { change from a } \\
\text { number of } \\
\text { intervention } \\
\text { studies (e.g., Ando } \\
\text { et al., 2010; } \\
\text { Breitbart et al., } \\
\text { 2012; 2010; Henry } \\
\text { et al., 2010) }\end{array}$ \\
\hline “ & $\begin{array}{l}\text { ICQ (Evers et al., } \\
\text { 2001) }\end{array}$ & $\begin{array}{l}\text { Administration: self- } \\
\text { report } \\
\text { Items: } 18 \\
\text { Response scale: } \\
\text { 4-point }(0=\text { not at } \\
\text { all to } \\
4=\text { completely) } \\
\text { Scores: } \\
\text { range for each } 6- \\
\text { item scale } 0-24\end{array}$ & & $\begin{array}{l}\text { Helplessness } \\
\quad(6 \text { items }) \\
\alpha=0.88 \\
\text { Acceptance } \\
(6 \text { items }) \\
\alpha=0.90-0.91 \\
\text { Perceived benefits } \\
(6 \text { items }) \\
\alpha=0.84-0.85\end{array}$ & $\begin{array}{l}\text { Test-retest reliability (1 } \\
\quad \text { year, } n=81 \text { RA, } n=67 \\
\text { MS) } \\
r=0.68 \text { to } 0.79\end{array}$ & + & $?$ \\
\hline “ & $\begin{array}{l}\text { ICS (Kroz et al., } \\
2009)\end{array}$ & $\begin{array}{l}\text { Administration: self- } \\
\text { report } \\
\text { Items: } 10 \\
\text { Response scale: } \\
\text { 5-point (1-5) } \\
\text { Scores: } \\
\text { range } 10-50\end{array}$ & $\begin{array}{l}57 \text { cancer patients; } 57 \\
\text { matching controls } \\
\text { (a) } 17 \text { patients } \\
\text { with breast cancer } \\
\text { and } 25 \text { with } \\
\text { colorectal cancer } \\
\text { receiving chemo } \\
\text { (b) Germany }\end{array}$ & $\begin{array}{l}\text { Inner resilience \& coherence } \\
\quad(8 \text { items }) \\
\alpha=0.91 \\
\text { Range } 8-40 \\
\text { Thermo-coherence } \\
(2 \text { items }) \\
\alpha=0.85 \\
\text { Range } 2-10\end{array}$ & $\begin{array}{l}\text { Internal consistency: } \\
\quad \alpha=0.91 \\
\text { Test-retest } \\
\quad(n=65 ; \text { median } \\
4 \text { weeks) } \\
r=0.80 \text { (a) }\end{array}$ & + & + \\
\hline
\end{tabular}




\begin{tabular}{|c|c|c|c|c|c|c|}
\hline $\begin{array}{l}\text { LAP (Reker \& } \\
\quad \text { Peacock, 1981) }\end{array}$ & $\begin{array}{l}\text { Administration: self- } \\
\text { report } \\
\text { Items: } 56 \\
\text { Response scale: } \\
7 \text { point } \\
(1=\text { strongly } \\
\text { disagree to } \\
7=\text { strongly agree }) \\
\text { Scores: } \\
\text { Range unclear }\end{array}$ & & $\begin{array}{l}\text { Life purpose } \\
\quad(9 \text { items }) \\
\alpha=0.83 \\
\text { Existential } \\
\text { vacuum } \\
(7 \text { items }) \\
\alpha=0.75 \\
\text { Life control } \\
(6 \text { items }) \\
\alpha=0.78 \\
\text { Death acceptance } \\
(6 \text { items }) \\
\alpha=0.70 \\
\text { Will to meaning } \\
(6 \text { items }) \\
\alpha=0.57 \\
\text { Goal seeking } \\
(5 \text { items }) \\
\alpha=0.66 \\
\text { Future meaning } \\
\text { to fulfill } \\
\text { (5 items) } \\
\alpha=0.55\end{array}$ & $\begin{array}{l}\text { Subscales } \\
\qquad \alpha=0.55-0.83\end{array}$ & + & ? \\
\hline LAP-R (Reker, 1992) & $\begin{array}{l}\text { Administration: self- } \\
\text { report } \\
\text { Items: } 48 \\
\text { Response scale: } \\
7 \text { point } \\
\text { (1= strongly } \\
\text { disagree to } \\
7=\text { strongly agree) } \\
\text { Scores: } \\
\text { Personal Meaning } \\
\text { Index ( } 16 \text { items) } \\
\text { Range } 16-112\end{array}$ & Various & $\begin{array}{l}\text { Life purpose } \\
\text { Coherence } \\
\text { Choice/responsibleness } \\
\text { Death acceptance } \\
\text { Existential vacuum } \\
\text { Goal seeking } \\
\text { Composite scores: } \\
\text { Personal Meaning Index } \\
\text { Existential transcendence }\end{array}$ & $\begin{array}{l}\text { Internal consistency: } \\
\quad \alpha=0.77 \text { to } 0.91 \\
\text { Test-retest reliability: } \\
\text { (4-6 weeks) } \\
r=0.77 \text { to } 0.90\end{array}$ & + & ? \\
\hline $\begin{array}{l}\text { LEQ (Salmon et al., } \\
1996 \text { ) }\end{array}$ & $\begin{array}{l}\text { Administration: self- } \\
\text { report } \\
\text { Items: } 61 \\
\text { Response scale: } \\
\text { 7-point scale with } \\
\text { opposing items at } \\
\text { end of each scale } \\
\text { (0-6) } \\
\text { Scoring not } \\
\text { specified }\end{array}$ & $\begin{array}{l}201 \text { patients with } \\
\text { incurable cancer. } \\
\text { U.K. }\end{array}$ & $\begin{array}{l}\text { Freedom } \\
\quad \alpha=0.70 \\
\text { Range } 0-60 \\
\text { Appreciation of life, } \\
\alpha=0.76 \\
\text { Range } 0-48 \\
\text { Contentment } \\
\alpha=0.76 \\
\text { Range } 0-54 \\
\text { Resentment } \\
\alpha=0.85 \\
\text { Range } 0-78 \\
\text { Social integration } \\
\alpha=0.78 \\
\text { Range } 0-48\end{array}$ & $\begin{array}{l}\text { Test-retest reliability } \\
\quad(n=40 ; 48-72 \mathrm{hrs}) \\
\quad r=0.77 \text { to } 0.92\end{array}$ & ? & ? \\
\hline
\end{tabular}




\begin{tabular}{|c|c|c|c|c|c|c|c|}
\hline Construct Measured & Tool & Description & Cancer Validation* & Domains & Reliability & $\begin{array}{l}\text { Construct Validity } \\
(+/ ? /-)^{* * *}\end{array}$ & $\begin{array}{l}\text { Responsiveness to } \\
\text { Change }(+/ ? /-)^{* * *}\end{array}$ \\
\hline “ & $\begin{array}{l}\text { Meaning in Life } \\
\text { questions (Tomich } \\
\text { \& Helgeson, 2002) }\end{array}$ & $\begin{array}{l}\text { Administration: self- } \\
\text { report } \\
\text { Items: } 20 \\
\text { Response scale: } \\
\text { 4-point ( } 1=\text { none, } \\
4=\text { a lot) for first } 3 \\
\text { domains; } 5 \text { point } \\
\text { (1= not at all; } \\
5=\text { very much) for } \\
\text { remaining } 2 \\
\text { domains }\end{array}$ & $\begin{array}{l}164 \text { breast cancer } \\
\text { survivors and } 164 \\
\text { age-matched } \\
\text { controls. } \\
\text { United States }\end{array}$ & $\begin{array}{l}\text { Search for meaning } \\
\text { (2 items) } \\
\text { Benefit } \\
(1 \text { item }) \\
\text { Harm } \\
(1 \text { item }) \\
\text { Personal growth } \\
\text { (9 items) } \\
\text { Acceptance } \\
\text { (7 items) }\end{array}$ & $\begin{array}{l}\text { Internal consistency: } \\
\alpha=0.86 \text { to } 0.89 \text { for } \\
\text { personal growth and } \\
\text { acceptance (used in } \\
\text { subsequent research as } \\
\text { the "Benefit Finding } \\
\text { Scale") }\end{array}$ & $?$ & $\begin{array}{l}\text { + (Antoni et al., 2001; } \\
\text { Chandwani et al., } \\
\text { 2010; Penedo et al., } \\
\text { 2006) }\end{array}$ \\
\hline “ & $\begin{array}{l}\text { MLQ (Steger et al., } \\
\text { 2006) }\end{array}$ & $\begin{array}{l}\text { Administration: self- } \\
\text { report } \\
\text { Items: } 10 \\
\text { Response scale: } 7- \\
\text { point } \\
\text { (1=absolutely } \\
\text { untrue to } \\
7=\text { absolutely } \\
\text { true })\end{array}$ & & $\begin{array}{l}\text { Presence } \\
\quad \text { (5 items) } \\
\alpha=0.86 \\
\text { Range 7-35 } \\
\text { Search } \\
\text { (5 items) } \\
\alpha=0.87 \\
\text { Range 7-35 }\end{array}$ & $\begin{array}{l}\text { See individual scale scores } \\
\quad \text { Target self-reports } \\
\alpha=0.81-0.84 \\
\text { Test-retest reliability } \\
(n=70 ; 1 \text { month }) \\
r=0.70-0.73\end{array}$ & + & $\begin{array}{l}\text { ? Some evidence for } \\
\text { responsiveness to } \\
\text { change of MLQ- } \\
\text { Search subscale } \\
\text { (Hsiao et al., 2012) }\end{array}$ \\
\hline “ & $\begin{array}{l}\text { MiLS (Jim et al., } \\
\text { 2006) }\end{array}$ & $\begin{array}{l}\text { Administration: self- } \\
\text { report } \\
\text { Items: } 21 \\
\text { Response scale: } \\
6 \text {-pt ( } 14 \text { items }) \\
\text { (1= strongly } \\
\text { disagree to } \\
6=\text { strongly agree) } \\
5 \text {-pt ( } 7 \text { items) } \\
(0=\text { not at all to } \\
4=\text { very much) } \\
\text { Scores: } \\
\text { Each scale scored } \\
\text { to have a range of } \\
1-6 \\
\text { Total calculated as } \\
\text { scores for } 3 \text { positive } \\
\text { scales minus score } \\
\text { for "confusion and } \\
\text { lessened meaning"; } \\
\text { possible range } \\
\text { of }-3 \text { to } 17\end{array}$ & $\begin{array}{l}167 \text { survivors of } \\
\text { breast cancer at } \\
\text { least } 2 \text { years post- } \\
\text { diagnosis (a); } \\
384 \text { survivors of } \\
\text { mixed cancers } \\
\text { recruited via the } \\
\text { internet (b) }\end{array}$ & $\begin{array}{l}\text { Harmony and peace ( } 4 \text { items) } \\
\quad \alpha=0.87 \\
\text { Life perspective, purpose } \\
\text { and goals ( } 7 \text { items) } \\
\alpha=0.90 \\
\text { Confusion and lessened } \\
\text { meaning } \\
\text { ( } 7 \text { items) } \\
\alpha=0.84 \\
\text { Benefits of spirituality } \\
\text { (3 items) } \\
\alpha=0.91\end{array}$ & $\begin{array}{l}\text { Internal consistency: } \\
\quad \alpha=0.93 \\
\text { Test-retest: } \\
\quad(n=43,2 \text { weeks }) \\
r=0.80 \\
\text { (0.67 to } 0.81 \text { for } \\
\text { subscales) }\end{array}$ & + & $?$ \\
\hline “ & MIST (Starck, 1983) & $\begin{array}{l}\text { Administration: self- } \\
\text { report } \\
\text { Items: } 20 \\
\text { Response scale: } \\
7 \text {-pt }(1=\text { never to } \\
7=\text { constantly }) \\
\text { Scores: } \\
20-140\end{array}$ & & $\begin{array}{l}\text { Subjective characteristics of } \\
\text { suffering } \\
\text { (6 items) } \\
\text { Personal responses to } \\
\text { suffering (8 items) } \\
\text { Meaning of suffering } \\
\text { (6 items) }\end{array}$ & $\begin{array}{l}\text { Internal consistency: } \\
\text { Total: } \alpha=0.81 \text { to } 0.83 \\
\text { Subscales: } \\
\alpha=0.52 \text { to } 0.74 \\
\text { Split half: } r=0.82 \\
\text { (Melton \& Schulenberg, } \\
\text { 2008) }\end{array}$ & $\begin{array}{l}+ \text { (Melton \& } \\
\text { Schulenberg, 2008) }\end{array}$ & $?$ \\
\hline
\end{tabular}




$\begin{array}{cl}\text { PMCPI (Chen, 1999) } & \text { Administration: self- } \\ & \text { report } \\ & \text { Items: } 27 \\ & \text { Response scale: } \\ & 5 \text {-pt }(1=\text { this is not } \\ & \text { like my thought at } \\ & \text { all to } 5=\text { this is } \\ & \text { exactly my } \\ \text { thought) } & \text { Scoring not } \\ \text { specified }\end{array}$
Profile (Wong,

\section{Administration: self- report
Items: 57 \\ Response scale: \\ 7 -pt $(1=$ not at all} to $7=$ a great deal

$\begin{array}{ll}\begin{array}{c}\text { Positive Meaning and } \\ \text { Vulnerability }\end{array} & \begin{array}{c}\text { Administration: self- } \\ \text { Scale (Bower et al., }\end{array} \\ \text { 2005) } & \text { Items: } 11 \\ & \text { Response scale: } \\ & 5 \text {-pt }(0=\text { not at all } \\ & \text { to } 4=\text { very much) } \\ & \\ & \\ & \\ & \\ \text { PIL (Crumbaugh \& } & \text { Administration: self- } \\ \text { Maholick, 1964) } & \text { report } \\ & \text { Items: } 20 \\ & \text { Response scale: } \\ & \text { 7-pt } \\ & \text { Range: } 20-140\end{array}$
patients, all a least 1 year

post diagnosis. The Netherland
(Jaarsma et al., (Jaarsma et al.,

00 cancer patients
who were
experiencing pain.
Taiwan

94 mixed cancer
patients, all at
least 1 year
post diagnosis.
The Netherlands
(Jaarsma et al.,
2007)

nitial factor analysis on T1 scores of 826 disease-free breast confirmatory

factor analysis on

T1 scores of 1088 disease-free breast 763 disease-free breast cancer

survivors

completed

questionnaires at

questionnaires at
both T1 and T2 (on

average 2.8 years

later)

None specified

(4 items)

Blame other

(4 items)

Blame self

(5 items)

(9 items)

Achievemen

(16 items)

Relationship

(9 items)

(8 items)

(6 items)

Intimacy

(4 items)

(6 items)

(5 items)
Subscales varied from

$\alpha=0.55$ to 0.81

$\alpha=0.72-0.80$

Threat (5 items)

$\alpha=0.76-0.81$

$\alpha=0.66-0.75$

$\alpha=0.55-0.56$

$(4$ items $)$
$\alpha=0.57-0.67$

$\alpha=0.57-0.67$
Spiritual awareness

$\alpha=0.75-0.76$

Self-transcendence

Self-acceptance

Fair treatment

Positive meaning

$\alpha=0.84$
Vulnerability

$(5$ items)
$\alpha=0.81$ to 0.83 


\begin{tabular}{|c|c|c|c|c|c|c|c|}
\hline Construct Measured & Tool & Description & Cancer Validation* & Domains & Reliability & $\begin{array}{l}\text { Construct Validity } \\
(+/ ? /-)^{* * *}\end{array}$ & $\begin{array}{l}\text { Responsiveness to } \\
\text { Change }(+/ ? /-)^{* * *}\end{array}$ \\
\hline " & $\begin{array}{l}\text { PUB (Passik et al., } \\
\text { 2003) }\end{array}$ & $\begin{array}{l}\text { Administration: self- } \\
\text { report } \\
\text { Items: } 14 \\
\text { Response scale: } \\
4 \text { pt }(1=\text { none of } \\
\text { the time to } 4=\text { all } \\
\text { of the time) } \\
\text { Range: } 14-56\end{array}$ & $\begin{array}{l}100 \text { cancer patients } \\
\text { with mixed cancer } \\
\text { types } \\
\text { United States }\end{array}$ & $\begin{array}{l}\text { Overt boredom } \\
\quad(8 \text { items }) \\
\alpha=0.93 \\
\text { Boredom related to } \\
\text { meaning and spirituality } \\
(6 \text { items }) \\
\alpha=0.85\end{array}$ & $\begin{array}{l}\text { Internal consistency: } \\
\quad \alpha=0.84 \\
\text { Test-retest reliability } \\
(n=20,7 \text { days): } \\
\quad r=0.80\end{array}$ & + & $?$ \\
\hline " & $\begin{array}{l}\text { SMiLE (Fegg et al., } \\
\text { 2008) }\end{array}$ & $\begin{array}{l}\text { Administration: self- } \\
\text { report } \\
\text { Items: } 3-7 \text { self- } \\
\text { nominated areas } \\
\text { providing meaning } \\
\text { to life rated for } \\
\text { current importance } \\
\text { and satisfaction } \\
\text { Response scale: } \\
\text { 7-pt; satisfaction } \\
\text { (-3= very } \\
\text { unsatisfied to } \\
3=\text { very satisfied); } \\
\text { importance } \\
(0=\text { not important } \\
\text { to } 7=\text { extremely } \\
\text { important) } \\
\text { Scores: indices of } \\
\text { total weighting } \\
\text { (IoW, 20-100), } \\
\text { total satisfaction } \\
\text { (IoS, } 0-100 \text { ); total } \\
\text { weighted } \\
\text { satisfaction (IoWS, } \\
0-100)\end{array}$ & $\begin{array}{l}599 \text { students in } \\
\text { Munich and } \\
\text { Dublin and } 75 \\
\text { palliative care } \\
\text { patients in } \\
\text { Munich (majority } \\
\text { cancer). } \\
\text { Germany, Ireland }\end{array}$ & $\begin{array}{l}\text { Respondent-nominated } \\
\text { satisfaction } \\
\alpha=0.71 \\
\text { Importance } \\
\alpha=0.49\end{array}$ & $\begin{array}{l}\text { Test-retest } \\
\text { (7 days): } \\
\text { IoW } 0.60 \\
\text { IoS } 0.71 \\
\text { IoWS } 0.72 \\
85.6 \% \text { of items listed at } \\
\text { T1 were listed again at } \\
\text { T2 }\end{array}$ & + & 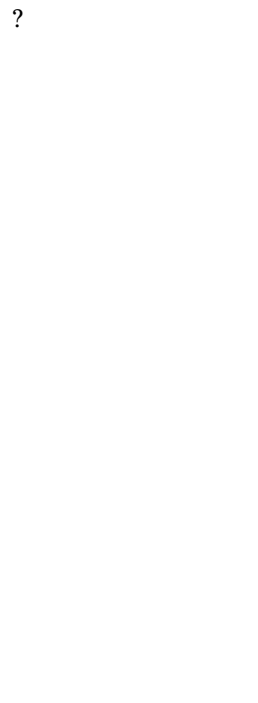 \\
\hline " & $\begin{array}{l}\text { Sense of Coherence } \\
\text { Scale (Orientation } \\
\text { to Life } \\
\text { Questionnaire) } \\
\text { (Antonovsky, } \\
\text { 1993) }\end{array}$ & $\begin{array}{l}\text { Administration: self- } \\
\text { report } \\
\text { Items: } 29 \\
\text { (13-item version } \\
\text { also available) } \\
\text { Response scale: } \\
\text { 7-pt semantic } \\
\text { differential } \\
\text { (different anchors } \\
\text { for each question) } \\
\text { Range: } \\
\text { 13-91 (SOC-13) } \\
\text { 29-203 (SOC-29) }\end{array}$ & & $\begin{array}{l}\text { Comprehensibility (11 items) } \\
\text { Manageability } \\
\text { (10 items) } \\
\text { Meaningfulness } \\
\text { (8 items) }\end{array}$ & $\begin{array}{l}\text { Internal consistency: } \\
\text { SOC- } 29 \\
\alpha=0.82-0.95 \\
\text { SOC }-13 \\
\alpha=0.74-0.91 \\
\text { Test-retest: } \\
r=0.41-0.91 \\
(2 \text { weeks to } 2 \text { years })\end{array}$ & + & $\begin{array}{l}\text { ? Some evidence of } \\
\text { responsiveness to } \\
\text { change from } \\
\text { studies evaluating } \\
\text { interventions } \\
\text { (Delbar \& Benor } \\
\text { Dan, 2001; } \\
\text { Henderson et al., } \\
\text { 2012) }\end{array}$ \\
\hline
\end{tabular}




\begin{tabular}{|c|c|c|c|c|c|c|c|}
\hline “ & $\begin{array}{l}\text { SOMP and SOMP-R } \\
\quad \text { (Reker, 1996) }\end{array}$ & $\begin{array}{l}\text { Administration: self- } \\
\text { report } \\
\text { Items: } 17 \\
\text { Response scale: } \\
\text { 7-pt (not at all } \\
\text { meaningful to } \\
\text { extremely } \\
\text { meaningful) } \\
\text { Range: } \\
\text { Total } \\
\text { "Breadth" score } \\
\text { (number of items } \\
\text { scored at greater } \\
\text { than or equal to 5) }\end{array}$ & & $\begin{array}{l}\text { Self-transcendence } \\
\text { Collectivism } \\
\text { Individualism } \\
\text { Self-preoccupation }\end{array}$ & $\begin{array}{l}\text { Internal consistency: } \\
\quad \alpha=0.71 \text { to } 0.80 \\
\text { Test-retest reliability: } \\
(3 \text { month }) \\
r=0.70\end{array}$ & + & $?$ \\
\hline “ & $\begin{array}{l}\text { World Assumptions } \\
\text { Scale (Janoff- } \\
\text { Bulman, 1989) }\end{array}$ & $\begin{array}{l}\text { Administration: self- } \\
\text { report } \\
\text { Items: } 32 \\
\text { Response scale: } \\
\text { 8-pt (disagree } \\
\text { completely to agree } \\
\text { completely) } \\
\text { Subsequent work } \\
\text { also done with 6-pt } \\
\text { scale (strongly } \\
\text { agree to strongly } \\
\text { disagree) } \\
\text { Range: scores } \\
\text { totaled for each } \\
\text { subscale }\end{array}$ & & $\begin{array}{l}\text { Justice } \\
\text { Controllability } \\
\text { Randomness } \\
\text { Self-worth } \\
\text { Self-controllability } \\
\text { Luck } \\
\text { Benevolence of people and } \\
\text { benevolence of the } \\
\text { impersonal world } \\
\text { (emerged as one factor } \\
\text { rather than two) }\end{array}$ & $\begin{array}{l}\text { Subscale reliabilities } \\
\text { between } 0.66 \text { and } 0.78\end{array}$ & $?$ & $?$ \\
\hline Spiritual Well-Being & $\begin{array}{l}\text { Are you at peace? } \\
\text { Single item (Stein- } \\
\text { hauser et al., 2006) }\end{array}$ & $\begin{array}{l}\text { Administration: self- } \\
\text { report } \\
\text { Items: } 1 \\
\text { Response scale: } \\
\text { 5-point }\end{array}$ & $\begin{array}{l}248 \text { patients with } \\
\text { advanced serious } \\
\text { illness }(56 \% \\
\text { cancer) }\end{array}$ & Single question & $\begin{array}{l}\alpha=\mathrm{n} / \mathrm{a} \\
\text { No test-retest reliability } \\
\text { reported }\end{array}$ & + & 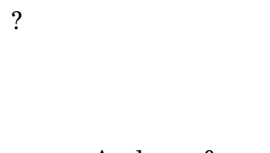 \\
\hline “ & $\begin{array}{l}\text { FACIT-Sp } \\
\text { (Peterman, et al., } \\
\text { 2002) (a) } \\
\text { 3-factor (Canada } \\
\text { et al., 2008) (b) }\end{array}$ & $\begin{array}{l}\text { Administration: self- } \\
\text { report } \\
\text { Items: } 12 \\
\text { Response scale: } \\
\text { 5-point }(0=\text { not at } \\
\text { all to } 4=\text { very })\end{array}$ & $\begin{array}{l}\text { 1,167 patients ( } 83 \% \\
\text { with cancer) (a) } \\
240 \text { long-term } \\
\text { female cancer } \\
\text { survivors (b) } \\
8805 \text { cancer } \\
\text { survivors (c), } \\
\text { United States }\end{array}$ & $\begin{array}{l}\text { Sense of meaning } \\
\text { Sense of peace } \\
\text { Role of faith in illness }\end{array}$ & $\begin{array}{c}\alpha=0.87(\mathrm{a}) \\
\alpha=0.85(\mathrm{~b}) \\
\alpha=0.88(\mathrm{c})\end{array}$ & + & $\begin{array}{l}\text { + e.g., Axelsson \& } \\
\text { Sjoden, 1999; } \\
\text { Byock \& } \\
\text { Merriman, 1998; } \\
\text { Henoch et al., 2010; } \\
\text { Selman et al., 2011) }\end{array}$ \\
\hline “ & $\begin{array}{l}\text { JAREL } \\
\text { (Hungelmann } \\
\text { et al., 1996) }\end{array}$ & $\begin{array}{l}\text { Administration: self- } \\
\text { report } \\
\text { Items }=21 \\
\text { Response scale: } \\
\text { 6-point (strongly } \\
\text { agree to strongly } \\
\text { disagree) }\end{array}$ & & $\begin{array}{l}\text { Faith/belief } \\
\text { Life/self-responsibility } \\
\text { Life-satisfaction/self- } \\
\text { actualization }\end{array}$ & $\begin{array}{l}\text { Internal consistency: } \\
\qquad \alpha=0.85\end{array}$ & $?$ & $?$ \\
\hline “ & $\begin{array}{l}\text { LASA (Johnson et al., } \\
\text { 2007) (a) (Locke } \\
\text { et al., 2007) (b) }\end{array}$ & $\begin{array}{l}\text { Administration: self- } \\
\text { report } \\
\text { Items: } 1 \\
\text { Response scale: } \\
100-\mathrm{mm} \text { line from } \\
0=\text { as bad as it can } \\
\text { be to } 10=\text { as good } \\
\text { as it can be }\end{array}$ & $\begin{array}{l}\text { (a) } 103 \text { patients with } \\
\text { advanced cancer } \\
\text { receiving radiation } \\
\text { therapy. } \\
\text { United States } \\
\text { (b) } 205 \text { patients } \\
\text { with newly } \\
\text { diagnosed high- } \\
\text { grade glioma. } \\
\text { United States }\end{array}$ & Single item & $\begin{array}{l}\text { Test-retest not reported } \\
\text { Internal consistency: } \mathrm{n} / \mathrm{a}\end{array}$ & + & $?$ \\
\hline
\end{tabular}




\begin{tabular}{|c|c|c|c|c|c|c|c|}
\hline Construct Measured & Tool & Description & Cancer Validation* & Domains & Reliability & $\begin{array}{l}\text { Construct Validity } \\
(+/ ? /-)^{* * *}\end{array}$ & $\begin{array}{l}\text { Responsiveness to } \\
\text { Change }(+/ ? /-)^{* *}\end{array}$ \\
\hline “ & $\begin{array}{l}\text { PEACE (Mack et al., } \\
\text { 2008) }\end{array}$ & $\begin{array}{l}\text { Administration: self- } \\
\text { report } \\
\text { Items: } 12 \\
\text { Response scale: } \\
\text { 4-point }(1=\text { not at } \\
\text { all to } 4=\text { to a large } \\
\text { extent) }\end{array}$ & $\begin{array}{l}160 \text { patients with } \\
\text { advanced cancer } \\
\text { and failure of first- } \\
\text { line chemo. } \\
\text { United States }\end{array}$ & $\begin{array}{l}\text { Struggle with illness } \\
\text { Peaceful acceptance }\end{array}$ & $\begin{array}{l}\text { Internal consistency: } \\
\text { Subscale } 1 \alpha=0.81 \\
\text { Subscale } 2 \alpha=0.78\end{array}$ & $+?$ & $?$ \\
\hline " & $\begin{array}{l}\text { STS } \\
\text { (a) (Reed, 1991) } \\
\text { (b) (Thomas et al., } \\
2010 \text { ) }\end{array}$ & $\begin{array}{l}\text { Administration: self- } \\
\text { report } \\
\text { Items: } 15 \\
\text { Response scale: } \\
4 \text {-point ( } 1=\text { not at } \\
\text { all to } 4=\text { very } \\
\text { much) }\end{array}$ & $\begin{array}{l}55 \text { older adult cancer } \\
\text { patients } \\
\text { (a) } \\
87 \text { women } \\
\text { diagnosed with } \\
\text { breast cancer in } \\
\text { the last } 5 \text { years (b) } \\
\text { United States }\end{array}$ & Single domain & $\begin{array}{l}\text { Internal consistency: } \\
\quad \alpha=0.8 \text { to } 0.93\end{array}$ & + & $?$ \\
\hline " & $\begin{array}{l}\text { Spirit } 8 \text { (Selman } \\
\text { et al., 2012) }\end{array}$ & $\begin{array}{l}\text { Administration: self- } \\
\text { report } \\
\text { Items: } 8 \\
\text { Response scale }=5 \text { - } \\
\text { point }(1=\text { worst to } \\
5=\text { best })\end{array}$ & $\begin{array}{l}285 \text { palliative care } \\
\text { patients, } 18 \% \\
\text { cancer } \\
\text { Africa }\end{array}$ & Single domain & $\begin{array}{l}\text { Internal consistency: } \\
\quad \alpha=0.73\end{array}$ & $?$ & $?$ \\
\hline " & SHI (Highfield, 1992) & $\begin{array}{l}\text { Administration: self- } \\
\text { report form } \\
\text { Nurse report form } \\
\text { Items: } 31 \\
\text { Response scale: } \\
5 \text {-point ( } 1=\text { never } \\
\text { to } 5=\text { all of the } \\
\text { time) }\end{array}$ & $\begin{array}{l}23 \text { patients with } \\
\text { primary lung } \\
\text { cancer and } 27 \\
\text { registered nurses } \\
\text { caring for them in } \\
2 \text { religiously } \\
\text { affiliated } \\
\text { hospitals. } \\
\text { United States }\end{array}$ & $\begin{array}{l}\text { Spiritual needs for: } \\
\text { Self-acceptance } \\
\text { Relationships } \\
\text { Hope }\end{array}$ & $\begin{array}{l}\text { Internal consistency: } \\
\text { Patient form: } \alpha=0.77 \\
\text { Nurse form: } \alpha=0.89\end{array}$ & $?$ & $?$ \\
\hline “ & SPS (Reed, 1987) & $\begin{array}{l}\text { Administration: self- } \\
\text { report } \\
\text { Items: } 10 \\
\text { Response scale: } \\
\text { 6-point (response } \\
\text { options vary } \\
\text { between items) }\end{array}$ & $\begin{array}{l}100 \text { terminally ill } \\
\text { hospitalized } \\
\text { cancer patients. } \\
\text { United States }\end{array}$ & Single domain & $\begin{array}{l}\text { Internal consistency: } \\
\alpha=0.93-0.95 \\
\text { across groups }\end{array}$ & + & $?$ \\
\hline “ & $\begin{array}{l}\text { STM (Leung et al., } \\
\text { 2006) }\end{array}$ & $\begin{array}{l}\text { Administration: self- } \\
\text { report } \\
\text { Items: } 22 \\
\text { Response scale: } \\
\text { 5-point (5= highly } \\
\text { satisfied to } \\
1=\text { highly } \\
\text { unsatisfied) }\end{array}$ & $\begin{array}{l}37 \text { terminal cancer } \\
\text { patients admitted } \\
\text { to hospices. } \\
\text { Taiwan }\end{array}$ & $\begin{array}{l}\text { Situational transcendence } \\
\text { Moral transcendence } \\
\text { Religious transcendence }\end{array}$ & $\begin{array}{l}\text { Internal consistency: } \\
\quad \alpha=0.95\end{array}$ & + & $?$ \\
\hline “ & $\begin{array}{l}\text { SWBS } \\
\text { (a) (Ellison, 1983) } \\
\text { (b) (Sherman } \\
\text { et al., 2005) }\end{array}$ & $\begin{array}{l}\text { Administration: self- } \\
\text { report } \\
\text { Items: } 20 \\
\text { Response scale: } \\
\text { 6-point (strongly } \\
\text { agree to strongly } \\
\text { disagree) }\end{array}$ & $\begin{array}{l}\text { (b) } 38 \text { patients with } \\
\text { advanced cancer } \\
\text { and } 38 \text { caregivers } \\
\text { Also in this sample } \\
\text { were } 63 \text { patients } \\
\text { with advanced } \\
\text { AIDS patients and } \\
43 \text { of their carers, } \\
\text { reported } \\
\text { separately }\end{array}$ & $\begin{array}{l}\text { Religious well-being } \\
\text { Existential well-being } \\
\text { United States }\end{array}$ & $\begin{array}{l}\text { (a) Internal consistency: } \\
\alpha=0.89 \text { (SWB) } \\
\alpha=0.87 \text { (EWB) } \\
\text { Test-retest reliability: } \\
r=0.93 \text { (RWB) } \\
r=0.86 \text { (EWB) } \\
\text { (b) Internal consistency: } \\
\text { Patients } \\
\alpha=0.96 \text { (RWB) } \\
\alpha=0.78 \text { (EWB) } \\
\text { Carers } \\
\alpha=0.96 \text { (RWB) } \\
\alpha=0.81 \text { (EWB) }\end{array}$ & $?$ & $?$ \\
\hline
\end{tabular}




\begin{tabular}{|c|c|c|c|c|c|c|c|}
\hline $\begin{array}{l}\text { Multi- } \\
\text { dimensional } \\
\text { measures of } \\
\text { quality of life } \\
\text { including a } \\
\text { spiritual/ } \\
\text { existential } \\
\text { dimension }\end{array}$ & $\begin{array}{l}\text { HQLI } \\
\quad \text { (McMillan \& } \\
\quad \text { Weitzner, 1998) }\end{array}$ & $\begin{array}{l}\text { The HQLI was } \\
\text { developed } \\
\text { specifically for } \\
\text { hospice patients to } \\
\text { measure quality of } \\
\text { life. It has a } \\
\text { combined social/ } \\
\text { spiritual well-being } \\
\text { subscale. } \\
\text { Administration: } \\
\text { self-report } \\
\text { Items: } 8 \text { items in } \\
\text { subscale } \\
\text { Response options: } \\
0-10 \text { scale }\end{array}$ & $\begin{array}{l}294 \text { patients } \\
\text { with cancer } \\
\text { in hospices } \\
32 \text { healthy adults. } \\
\text { United States }\end{array}$ & Single domain (8 items) & $\begin{array}{l}\text { Internal consistency: } \\
\quad \alpha=0.82\end{array}$ & + & ? \\
\hline “ & $\begin{array}{l}\text { LTQL (Wyatt et al., } \\
\text { 1996) }\end{array}$ & $\begin{array}{l}\text { Developed to measure } \\
\text { QoL in long-term } \\
\text { female cancer } \\
\text { survivors. } \\
\text { The LTQL has a } \\
\text { spiritual/ } \\
\text { philosophical } \\
\text { subscale } \\
\text { Administration: } \\
\text { self-report } \\
\text { Items: } 8 \text { items in } \\
\text { subscale } \\
\text { Response scale: } \\
\text { 5-point Likert-type } \\
\text { scale }(0=\text { not at all, } \\
4=\text { very much })\end{array}$ & $\begin{array}{l}187 \text { female cancer } \\
\text { survivors } \\
\text { recruited through } \\
\text { the tumor registry. } \\
\text { United States }\end{array}$ & $\begin{array}{l}\text { Single domain: } \\
\text { Spiritual/philosophical }\end{array}$ & $\begin{array}{l}\text { Internal consistency: } \\
\quad \alpha=0.87\end{array}$ & + & $?$ \\
\hline “ & $\begin{array}{l}\text { MQoL (Cohen et al., } \\
\quad 1996 ; 1997)\end{array}$ & $\begin{array}{l}\text { Administration: self- } \\
\text { report } \\
\text { Total: } 16 \text { items } \\
\text { plus } 1 \text { global item } \\
\text { Existential well- } \\
\text { being subscale: } \\
6 \text { items } \\
\text { Response scale: } \\
0=\text { not at all to } \\
10=\text { extremely }\end{array}$ & $\begin{array}{l}247 \text { oncology day } \\
\text { centre patients. } \\
\text { Canada (Cohen } \\
\text { et al., 1996) } \\
143 \text { inpatients and } \\
\text { outpatients from } \\
\text { palliative care } \\
\text { services. } \\
\text { Canada (Cohen } \\
\text { et al., 1997) }\end{array}$ & $\begin{array}{l}\text { Physical symptoms } \\
\text { Psychological symptoms } \\
\text { Existential } \\
\text { well-being } \\
\text { Support }\end{array}$ & $\begin{array}{l}\text { Internal consistency: } \\
\quad \text { Total: } \alpha=0.83-0.89 \\
\text { (Cohen et al., } 1996 ; 1997) \\
\text { Existential: } \alpha=0.79- \\
0.87 \text { (Cohen et al., 1996; } \\
\text { 1997) } \\
\text { Test-retest reliability: } \\
\text { Total: } r=0.75 \text { (Cohen \& } \\
\text { Mount, 2000) } \\
\text { Existential: } \\
r=0.76 \text { (Cohen \& } \\
\text { Mount, 2000) }\end{array}$ & $+\underset{1997)}{(\text { Cohen et al., 1996; }}$ & $\begin{array}{l}+\underset{2000)}{(\text { Cohen \& Mount }}, \\
\quad\end{array}$ \\
\hline “ & $\begin{array}{l}\text { QHAL-EC (Lo et al., } \\
\text { 2011) }\end{array}$ & $\begin{array}{l}\text { Developed to measure } \\
\text { QoL in populations } \\
\text { near to the end of } \\
\text { life } \\
\text { Administration: } \\
\text { self-report } \\
\text { Items: } \\
\text { Prep for EoL }=5 \\
\text { items } \\
\text { Completion }=7 \\
\text { items } \\
\text { Response scale: } \\
\text { 5-item Likert-type } \\
\text { scale }\end{array}$ & $\begin{array}{l}464 \text { patients with } \\
\text { advanced cancer } \\
\text { from } 24 \text { outpatient } \\
\text { oncology clinics. } \\
\text { Canada }\end{array}$ & $\begin{array}{l}\text { Two domains: } \\
\text { Preparation for end of life } \\
\text { Life completion }\end{array}$ & $\begin{array}{l}\text { Internal consistency: } \\
\text { Preparation for end of } \\
\text { life: } \\
\alpha=0.73 \\
\text { Life completion: } \\
\alpha=0.83\end{array}$ & + & ? \\
\hline
\end{tabular}


Table 4. Continued

\begin{tabular}{|c|c|c|c|c|c|c|c|}
\hline Construct Measured & Tool & Description & Cancer Validation* & Domains & Reliability & $\begin{array}{l}\text { Construct Validity } \\
(+/ ? /-)^{* *}\end{array}$ & $\begin{array}{l}\text { Responsiveness to } \\
\text { Change }(+/ ? /-)^{* *}\end{array}$ \\
\hline “ & $\begin{array}{l}\text { QoLC-E (Pang et al., } \\
\text { 2005) }\end{array}$ & $\begin{array}{l}\text { Developed as a } \\
\text { measure of QoL of } \\
\text { Hong Kong } \\
\text { Chinese patients } \\
\text { with advanced } \\
\text { chronic disease }\end{array}$ & $\begin{array}{l}41 \text { metastatic cancer } \\
\text { patients. } \\
\text { Hong Kong }\end{array}$ & $\begin{array}{l}\text { Two domains: } \\
\text { Value of life ( } 6 \text { items) and } \\
\text { existential distress ( } 3 \\
\text { items) }\end{array}$ & $\begin{array}{l}\text { Internal consistency: } \\
\text { Value of life: } \alpha=0.83 \\
\text { Existential distress: } \\
\alpha=0.79\end{array}$ & + & $?$ \\
\hline " & $\begin{array}{l}\text { QoL-CS (Ferrell } \\
\text { et al., 1995) }\end{array}$ & $\begin{array}{l}\text { Administration: self- } \\
\text { report } \\
\text { Total: } 41 \text { items } \\
\text { Spiritual subscale: } \\
7 \text { items } \\
\text { Response scale: } \\
\text { Ordinal scale }\end{array}$ & $\begin{array}{l}686 \text { members of the } \\
\text { National Coalition } \\
\text { for Cancer } \\
\text { Survivorship } \\
\text { (NCCS). } \\
\text { United States }\end{array}$ & $\begin{array}{l}\text { Four domains: } \\
\text { Physical } \\
\text { Psychological } \\
\text { Social } \\
\text { Spiritual }\end{array}$ & $\begin{array}{l}\text { Internal consistency: } \\
\text { total: } \alpha=0.93 \\
\text { spiritual: } \alpha=0.71 \\
\text { Test-retest reliability } \\
\text { (two weeks): } \\
\text { total: } r=0.89 \\
\text { spiritual: } r=0.90\end{array}$ & + & $?$ \\
\hline “ & $\begin{array}{l}\text { QoL generic version } \\
\text { (Ferrans \& } \\
\text { Powers, 1985; } \\
\text { Ferrans et al., } \\
\text { 1992) } \\
\text { (QLI - CV) } \\
\text { (Ferrans, 1990) }\end{array}$ & $\begin{array}{l}\text { Administration: self- } \\
\text { report } \\
\text { Total: } 32 \text { items } \\
\text { Psychological/ } \\
\text { spiritual: } 7 \text { items } \\
\text { Response scale: } \\
6 \text { points } \\
\text { Part I: }(1=\text { very } \\
\text { dissatisfied } \\
6=\text { very satisfied }) \\
\text { Part II: }(1=\text { very } \\
\text { unimportant } \\
6=\text { very } \\
\text { important })\end{array}$ & $\begin{array}{l}111 \text { breast cancer } \\
\text { patients. } \\
\text { United States } \\
\text { (Ferrans, 1990) }\end{array}$ & $\begin{array}{l}\text { Four domains: } \\
\text { Health and functioning } \\
\text { Socioeconomic/ } \\
\text { psychological/ } \\
\text { spiritual } \\
\text { Family } \\
\text { Psychological/ } \\
\text { spiritual domain }\end{array}$ & $\begin{array}{l}\text { Internal consistency: } \\
\text { Total QLI: } \\
\alpha=0.90-0.95 \\
\text { (Ferrans, 1990; Ferrans } \\
\text { \& Powers, 1985; Ferrans } \\
\text { et al., 1992) } \\
\text { Test-retest reliability } \\
\text { (2 weeks): } \\
\text { Total QLI: } \\
r=0.81-0.87 \\
\text { (Ferrans \& Powers, } \\
\text { 1985) } \\
\text { Psychological/spiritual } \\
\text { domain } \\
\text { Internal consistency: } \\
\text { ( } \alpha=0.90-0.93 \text { ) } \\
\text { (Ferrans, 1990; Ferrans } \\
\& \text { Powers, 1992) } \\
\text { Test-retest reliability } \\
\text { not specified } \\
\text { (Ferrans \& Powers, } \\
\text { 1992) }\end{array}$ & $\begin{array}{l}\text { (Ferrans, 1990; } \\
\text { Ferrans \& Powers, } \\
\text { 1985; 1992) }\end{array}$ & $?$ \\
\hline “ & $\begin{array}{l}\text { SELT-M } \\
\text { (van Wegberg } \\
\text { et al., 1998) }\end{array}$ & $\begin{array}{l}\text { Developed to add a } \\
\text { spiritual dimension } \\
\text { to an existing } \\
\text { quality of life } \\
\text { measure. } \\
\text { Administration: } \\
\text { self-report } \\
\text { Items: } 8 \text { items in } \\
\text { subscale } \\
\text { Response scale: } \\
5 \text { point }(0=\text { not at } \\
\text { all to } 4=\text { entirely } \\
\text { so) }\end{array}$ & $\begin{array}{l}89 \text { patients with } \\
\text { metastatic, locally } \\
\text { advanced or } \\
\text { nonresectable } \\
\text { breast }(\mathrm{n}=49) \text { or } \\
\text { gastro-intestinal } \\
(\mathrm{n}=40) \text { cancer. } \\
\text { Switzerland }\end{array}$ & Single domain & $\begin{array}{l}\text { Internal consistency: } \\
\quad \alpha=0.73\end{array}$ & + & $?$ \\
\hline
\end{tabular}




\begin{tabular}{|c|c|c|c|c|c|c|}
\hline “ & $\begin{array}{l}\text { WHOQoL-100 SRPB } \\
\text { subscale } \\
\text { (a) (WHOQoL, } \\
2006 \text { ) } \\
\text { (b) (den Oudsten } \\
\text { et al., 2009) }\end{array}$ & $\begin{array}{l}\text { Developed as an } \\
\text { overarching } \\
\text { measure of quality } \\
\text { of life, the } \\
\text { WHOQoL-100 } \\
\text { covers } 24 \text { facets of } \\
\text { quality of life, The } \\
\text { SPRB scale was } \\
\text { developed to fully } \\
\text { measure spiritual } \\
\text { issues. } \\
\text { Administration: } \\
\text { self-report } \\
\text { Items: } 32 \\
\text { Response scale: } \\
\text { 5-point Likert-type } \\
\text { scale }\end{array}$ & $\begin{array}{l}\text { (b) } 356 \text { women with } \\
\text { breast } \\
\text { abnormality } \\
\text { (b) } 140 \text { breast } \\
\text { cancer survivors. } \\
\text { Holland }\end{array}$ & $\begin{array}{l}\text { Eight factors with four items } \\
\text { in each: spiritual } \\
\text { connection, meaning in } \\
\text { life, wholeness and } \\
\text { integration, spiritual } \\
\text { strength, inner peace, } \\
\text { hope and optimism and } \\
\text { faith. }\end{array}$ & $\begin{array}{l}\text { Internal consistency: } \\
\quad \alpha=0.91\end{array}$ & $+?$ \\
\hline $\begin{array}{l}\text { Spiritual pain, } \\
\text { distress and } \\
\text { struggle }\end{array}$ & $\begin{array}{l}\text { ELQ (Mayers et al., } \\
2002 \text { ) }\end{array}$ & $\begin{array}{l}\text { Administration: self- } \\
\text { report } \\
\text { Items: } 22 \\
\text { Response scale: } \\
\text { 6-point scale } \\
\text { (1= not at all true } \\
\text { of me, } 3= \\
\text { sometimes true of } \\
\text { me, } 6=\text { very much } \\
\text { true of me) } \\
\text { Scores: } \\
\text { range } 22-132\end{array}$ & & & $\begin{array}{l}\text { Internal consistency: } \\
\quad \alpha=0.90\end{array}$ & + \\
\hline 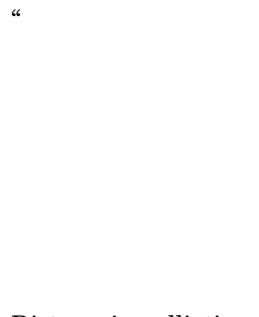 & $\begin{array}{l}\text { SDS [Taiwan] (Ku } \\
\text { et al., 2010) }\end{array}$ & $\begin{array}{l}\text { Administration: self- } \\
\text { report } \\
\text { Items: } 30 \\
\text { Response scale: } \\
\text { 4-point scale }(1-4) \\
\text { Scores: } \\
\text { range } 30-120 \\
\text { Higher scores } \\
\text { indicative of a } \\
\text { higher level of } \\
\text { spiritual distress }\end{array}$ & $\begin{array}{l}85 \text { cancer patients. } \\
\text { Taiwan }\end{array}$ & $\begin{array}{l}\text { Relations with self } \\
\text { Relations with others } \\
\text { Relations with God } \\
\text { Attitude toward death }\end{array}$ & $\begin{array}{l}\text { Internal consistency: } \\
\text { Total scale } \\
\alpha=0.95 \\
\text { Relations with self } \\
\alpha=0.93 \\
\text { Relations with others: } \\
\alpha=0.92 \\
\text { Relations with God: } \\
\alpha=0.90 \\
\text { Attitude toward death: } \\
\alpha=0.95\end{array}$ & 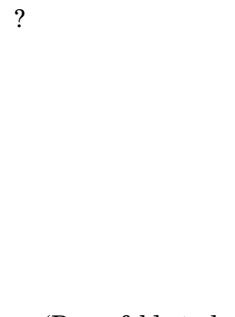 \\
\hline $\begin{array}{l}\text { Distress in palliative } \\
\text { care }\end{array}$ & $\begin{array}{l}\text { SAHD (Rosenfeld } \\
\text { et al., 1999; 2000) }\end{array}$ & $\begin{array}{l}\text { Administration: self- } \\
\text { report } \\
\text { Items: } 20 \\
\text { Response scale: } \\
\text { true/false } \\
\text { Scores: } \\
\text { range } 0-20 \\
\text { Higher scores } \\
\text { indicative of higher } \\
\text { level of desire for } \\
\text { death }\end{array}$ & $\begin{array}{l}92 \text { terminally ill } \\
\text { cancer patients } \\
\text { (life expectancy } \\
<6 \text { months). } \\
\text { USA (Rosenfeld } \\
\text { et al., 2000) }\end{array}$ & & $\begin{array}{l}\text { Internal consistency: } \\
\alpha=0.89 \text { (Rosenfeld } \\
\text { et al., 1999) } \\
\text { Internal consistency: } \\
\alpha=0.88 \text { (Rosenfeld } \\
\text { et al., 2000) } \\
\text { Split-half reliability: } \\
0.89\end{array}$ & $\begin{array}{c}+ \text { (Rosenfeld et al., } \\
2000 ; 1999)\end{array}$ \\
\hline
\end{tabular}




\begin{tabular}{|c|c|c|c|c|c|c|c|}
\hline Construct Measured & Tool & Description & Cancer Validation* & Domains & Reliability & $\begin{array}{l}\text { Construct Validity } \\
(+/ ? /-)^{* *}\end{array}$ & $\begin{array}{l}\text { Responsiveness to } \\
\text { Change }(+/ ? /-)^{* *}\end{array}$ \\
\hline “ & $\begin{array}{l}\text { SISC (Wilson et al., } \\
\text { 2004) }\end{array}$ & $\begin{array}{l}\text { Administration: } \\
\text { clinician } \\
\text { administered } \\
\text { Items: } 1 \\
\text { Response scale: } \\
7 \text {-point } \\
\text { (0= none to } \\
6=\text { extreme) } \\
1 \text { or } 2 \text { indicates the } \\
\text { experience of the } \\
\text { symptom or } \\
\text { concern is } \\
\text { relatively low. } \\
3 \text { corresponds to an } \\
\text { issue that is } \\
\text { generally a } \\
\text { significant } \\
\text { problem. Higher } \\
\text { scores associated } \\
\text { with clear presence } \\
\text { of symptom/ } \\
\text { concern at } \\
\text { clinically } \\
\text { important level- } \\
\text { varying degrees of } \\
\text { severity. }\end{array}$ & $\begin{array}{l}69 \text { palliative care } \\
\text { (advanced) cancer } \\
\text { patients. } \\
\text { Canada }\end{array}$ & Single item & $\begin{array}{l}\text { Interrater reliability: } \\
\quad \begin{array}{l}r=0.99 \\
\text { Test-retest ( } 1-3 \text { days): } \\
r=0.90\end{array}\end{array}$ & + & Not specified \\
\hline
\end{tabular}

*Key cancer validation sample (where applicable) reference and details listed.

$+=$ Bulk of the available evidence supportive of construct validity/responsiveness to change of the instrument, $-=$ bulk of available evidence does not support this property, ? = this property has not been assessed or contradictory results. 
Construct validity of the scale has been assessed in the context of dementia through correlations with comfort assessment in dying with dementia, but information on validity in the context of cancer is lacking at present. The scale appears responsive to change in the context of cancer, making it a potentially useful tool for monitoring patients and exploring the impact of interventions over time.

Clinician administration is both a strength and a limitation of this measure. It allows consistent assessment of all patients at the end of life, taking into account communication difficulties and avoiding burdening patients. However, clinician administration may also result in biased assessments, especially if clinicians responsible for the care of patients over time overestimate the impact of treatment.

\section{Pictorial Representation of Illness and Self Measure (PRISM, PRISM-R1 and PRISM-R2)}

The PRISM was originally intended as a measure of adjustment to illness, but qualitative analyses of content validity suggested its applicability as a measure of suffering (Büchi et al., 1998). The advantages of this measure include its brevity, simplicity, and ease of use (Büchi et al., 1998). In addition, by not specifying items and domains, it allows for a more subjective assessment of suffering due to illness, however patients might individually define this (Wouters et al., 2011; Wouters et al., 2008). Content validity has been explored in a number of qualitative studies (Büchi et al., 2002; Wouters et al., 2008), and there is evidence for reliability and validity (Büchi et al., 2002), although the lack of a gold-standard measure of suffering means that considerable work is necessary to satisfactorily validate this measure (Büchi et al., 2002). There are two revised versions of the measure, the PRISM-R1 and PRISM-R2 (Wouters et al., 2011), which provide additional information about the perceived severity of illness and incorporate a slightly revised response format.

The PRISM-R2 has been employed in the context of cancer survivorship, and evidence on the validity of the measure in this context has been presented (Wouters et al., 2011). The PRISM and its variants have been administered both face to face (Büchi et al., 2002; 1998; Wouters et al., 2008) and via mail (Wouters et al., 2011; Wouters et al., 2008), although it has been suggested that people with lower levels of education experienced some problems completing this more abstract measure, and face-to-face administration may be preferable (Wouters et al., 2011). The scale's developers also raise the possibility of administering the measure via computer (Büchi et al., 1998).

Considerable work has been done exploring the psychometric properties of the PRISM, and it shows some promise as a more subjective measure of suffering, perhaps especially in the context of face-to-face clinical work. Care should be taken in determining an appropriate mode of administration, and more work on definitional validity is recommended.

\section{Recommendation}

The PRISM has more evidence of validity and reliability than the MSSE, though more definitional clarity is required. Furthermore, it allows a nondirective approach and provides a quantitative score for serial assessment.

A single "Are you at peace?" item (Steinhauser et al., 2006) was identified as a measure of spiritual well-being and its validity assessed against other measures of spiritual well-being (see below). However, the authors referred to it as not only a measure of spiritual well-being but also a way of identifying suffering, so this measure should also be considered in the context of measures of suffering. Peace is one of the constructs measured by the FACIT-Sp (see below) in its three-factor version (Canada et al., 2008; Murphy et al., 2010) and could also be considered in this context.

\section{Hopelessness/Demoralization}

Seven instruments measuring hopelessness/demoralization were identified: the Beck Hopelessness Scale (BHS) (Beck et al., 1974); the despair subscale of the Cancer Care Monitor (CCM) (Fortner et al., 2003); Jacobsen et al.'s Demoralization Scale (Jacobsen et al., 2006); Kissane et al.'s (2004) Demoralization Scale; the Hopelessness Assessment in Illness (HAI) Questionnaire (Rosenfeld et al., 2011); a clinician-administered single-item screening instrument for hopelessness (Wilson et al., 2004); and the Subjective Incompetence Scale (SIS) (Cockram et al., 2009). The HAI and Kissane et al.'s Demoralization Scale are the most promising for assessing hopelessness and demoralization, respectively, in the advanced cancer context. Both, however, are relatively new measures and require further exploration of their psychometric properties. Other tools may be optimal depending on the research question.

\section{Hope}

Five measures assessing hope were identified. These included the Adult Dispositional Hope Scale (ADHS) (Snyder et al., 1991); the Herth Hope Scale (HHS)/ Herth Hope Index (HHI) (Herth, 1991; 1992); the Hope Differential (HD)/Hope Differential-Short (HDS) (Nekolaichuk \& Bruera, 2004; Nekolaichuk et al., 1999); Miller's Hope Scale (MHS) (Miller \& Powers, 1988); and the Nowotny Hope Scale (NHS) 
(Nowotny, 1989). Based on its brevity, frequency of use, and the availability of validation data in the cancer context, the HHI is optimal as a measure of hope. Note that all the measures listed have relatively high levels of internal consistency, implying that some items may be redundant.

\section{Meaning}

Some 20 scales were identified that measured meaning: the Chinese Cancer Coherence Scale (CCCS) (Chan et al., 2007); the Constructed Meaning Scale (Fife, 1995); the meaning/peace subscale of the Functional Assessment of Chronic Illness Therapy-Spiritual Well-Being Scale (FACIT-Sp) (Canada et al., 2008; Murphy et al., 2010; Peterman et al., 2002); the Illness Cognitions Questionnaire (ICQ) (Evers et al., 2001); the Internal Coherence Scale (ICS) (Kroz et al., 2009); the Life Attitude Profile (LAP)/ Life Attitude Profile-Revised (LAP-R) (Reker, 1992; Reker \& Peacock, 1981); the Life Evaluation Questionnaire (LEQ) (Salmon et al., 1996); the Meaning in Life questions (including the Benefit Finding Scale [BFS]) used by Tomich and Helgeson (2002); the Meaning in Life Questionnaire (MLQ) (Steger et al., 2006); the Meaning in Life Scale (MILS) (Jim et al., 2006); the Meaning in Suffering Test (MIST) (Starck, 1983); the Perceived Meanings of Cancer Pain Inventory (PMCPI) (Chen, 1999); the Personal Meaning Profile (PMP) (Wong, 1998); the Positive Meaning and Vulnerability Scale (Bower et al., 2005); the Purpose in Life (PIL) Test (Crumbaugh \& Maholick, 1964); the Purposelessness, Understimulation, and Boredom (PUB) Scale (Passik et al., 2003); the Schedule for Meaning in Life Evaluation (SMiLE) (Fegg et al., 2008); the Sense of Coherence (SOC) Scale (Antonovsky, 1993); the Sources of Meaning Profile (SOMP)/Sources of Meaning Profile-Revised (SOMP-R) (Reker, 1996); and the World Assumptions Scale (Janoff-Bulman, 1989). The optimal measure of meaning will vary depending on the purpose and context of the assessment. However, for assessing the spiritual dimension of global meaning, the FACIT-Sp should be considered optimal, and the LAP-R should be considered a strong candidate when exploring the relationship between global meaning and other variables. Optimal measures for assessing situational meaning will vary depending on the specific context and the construct to be assessed.

\section{Spiritual Well-Being}

A total of 11 measures assessing spiritual well-being were identified. These included a short "Are you at peace?" item (Steinhauser et al., 2006); the Functional Assessment of Chronic Illness Therapy-Spiritual
Well-Being Scale (FACIT-Sp) (Canada et al., 2008; Murphy et al., 2010; Peterman et al., 2002); the JAREL Spiritual Well-Being Scale (Hungelmann et al., 1996); a Linear Analogue Self-Assessment (LASA) item for spiritual well-being (Johnson et al., 2007; Locke et al., 2007); the Peace, Equanimity, and Acceptance in the Cancer Experience (PEACE) Scale (Mack et al., 2008); the Self-Transcendence Scale (STS) (Reed, 1991; Thomas et al., 2010); the Spirit 8 (Selman et al., 2012); the Spiritual Health Inventory (SHI) (Highfield, 1992); the Spiritual Perspective Scale (SPS) (Reed, 1987); the Spirituality Transcendence Measure (STM) (Leung et al., 2006); and the Spiritual Well-Being Scale (SWBS) (Ellison, 1983; Sherman et al., 2005). The FACIT-Sp may be optimal for assessing spiritual well-being in the cancer context. Its advantages include its development and validation in a large cancer population, its brevity, the frequency with which it is used in the context of cancer, and the substantive data available about its psychometric properties and to facilitate interpretation.

\section{Quality of Life}

Nine multidimensional measures of quality of life that included a spiritual/existential dimension were identified. These included the Hospice Quality of Life Index (HQLI) (McMillan \& Weitzner, 1998); the Long-Term Quality of Life (LTQL) instrument (Wyatt et al., 1996); the McGill Quality of Life (MQoL) Questionnaire (Cohen et al., 1997; 1996); the Quality of Life at the End of Life-Cancer (QUAL-EC) Scale (Lo et al., 2011); the Quality of Life Concerns in the End of Life (QoLC-E) Scale (Samantha et al., 2005); the Quality of Life for Cancer Survivors (QoL-CS) Scale (Ferrell, 1996); the Quality of Life Index (QLI)/Quality of Life Index-Cancer Version (QLI-CV) (Ferrans, 1990; Ferrans \& Powers, 1985, 1992); the Skalen zur Erfassung von Lebens Qualitat bei Tumorkranken-Modified Version (SELT-M) (van Wegberg et al., 1998); and the World Health Organization's Quality of Life Measure (WHOQoL-100) Spirituality/Religion/Personal Beliefs (SRPB) subscale (den Oudsten et al., 2009; WHOQoL, 2006). For multidimensional quality-oflife measurement incorporating an existential or spiritual domain, the MQoL questionnaire or FACIT-Sp appear optimal because substantive data are available about psychometric properties and interpretation in the cancer context.

\section{Distress in the Palliative Care Setting}

Two measures specifically assessing distress in the palliative care setting were identified: a clinician-administered single-item screening instrument for 
assessing desire for death, the Structured Interview for Symptoms and Concerns (SISC) (Wilson et al., 2004), and the Schedule of Attitudes toward Hastened Death (SAHD) (Rosenfeld et al., 2011; 1999). The latter questionnaire appears promising for assessing desire for death in the context of advanced cancer, though further validation in a larger sample is recommended.

\section{Pain, Distress, or Struggle of a Spiritual Nature}

Two measures assessing pain, distress, or struggle of a spiritual nature were identified: the Existential Loneliness Questionnaire (ELQ) (Mayers et al., 2002) and the Spiritual Distress Scale (SDS) (Ku et al., 2010). Further research validating these measures in larger cancer samples is necessary before either of these measures can be recommended.

\section{DISCUSSION}

Our review revealed that a number of instruments are suitable for measuring the various analogues of suffering but that challenges remain in this field, in part as a function of the complexity of suffering itself. Definitions of suffering and clear articulation of the aspects of suffering targeted by individual measures are essential. The multidimensional and individual nature of suffering should be taken into account when considering its assessment, as should its variance dependent on culture and context (Cassell, 1982; Wein, 2011). Many authors have noted the importance of context, including cultural, historical, and social factors that impact on the meaning an individual gives to an experience (Barton-Burke et al., 2008; Chio et al., 2008; Williams, 2004). Holistic assessment rather than a narrow focus on individual symptoms is recommended.

Further, it was evident from the review that information about the strengths, limitations, and psychometric properties of available measures for the specific use proposed should always be consulted when choosing an assessment tool. Such information will enable users to make an informed decision about the appropriate measure for any specific purpose, and/or may identify measures that might be further developed and assessed for validity. Lack of a goldstandard measure of suffering means that considerable work is necessary to satisfactorily validate these measures (Büchi et al., 2002).

Due to the individual nature of suffering and the manifold potential sources involved (Best et al., 2014), being able to assess both the personal elements of suffering for the patient as well as the objective would be advantageous. Measures to assess suffering may therefore be particularly useful if they include a subjective component (e.g., the PRISM, the SMiLE, the Hope Differential-Short, and the single item "Are you at peace?"); they may need to be supplemented by open questions and alertness to the nonverbal and verbal cues of the patient. Care should be taken in determining the appropriate mode of administration, and face-to-face administration would be considered preferable in populations with varying educational levels.

The distress of cancer patients who are suffering will often take a toll on their reserves that will make lengthy assessment tools burdensome in the clinical context. Further research should be conducted into the psychometric properties and usefulness of single-item measures of suffering, with potential use for clinical application as a screening tool and dooropener for discussion of patient concerns (Bayes et al., 1996). Potentially useful items include "Are you at peace?" and "How long did yesterday seem to you?" (Bayes et al., 1996).

Despite the numerous measures available for the assessment of suffering and its synonyms and symptoms, more work is needed to validate these tools in the cancer milieu. However, the wide range of assessment instruments currently under development will allow the clinician to focus on specific aspects of suffering to suit their clinical context.

\section{LIMITATIONS}

There are a number of limitations to the current review that should be acknowledged when interpreting results.

First, the search strategy adopted for our review was designed so as to allow for the synthesis of common elements across a number of concepts highlighted in the existing literature as potentially synonymous with suffering. Including existential and spiritual suffering/distress allowed due attention to be paid to an important and often overlooked dimension of suffering. The review authors believe that this broad synthesis of the common elements of these constructs enhances our understanding of the nature of suffering in the context of cancer; however, these concepts are not always seen as identical. The potentially useful nuances of each individual concept have therefore not been fully explored in this review.

Second, the search for measures focused on a list of "synonyms" and "symptoms" of suffering generated by an iterative review of the literature. This strategy allowed for the consistent inclusion of any measure targeting hope, meaning, or spiritual well-being; this appeared the most reliable and parsimonious of the possible search strategies identified. 
Third, holistic care in the cancer context involves not only the patient but also the family as the unit of care. The suffering of families and carers is deserving of attention; however, feasibility constraints precluded addressing this important issue within the scope of the current review.

\section{CONCLUSION}

This report reviews research published between 1992 and 2012 to identify validated tools that measure spiritual suffering or its symptoms in cancer patients. Some 90 articles were identified that yielded information about 58 measures. The constructs examined were: suffering, hopelessness/demoralization, hope, meaning, spiritual well-being and quality of life where a spiritual/existential dimension was included, distress in the palliative care setting, and pain, distress or struggle of a spiritual nature. The psychometric properties of these measures were compared. The PRISM shows promise as a direct measure of the burden of suffering due to illness, in terms of ease of use, and the possibility of capturing individual aspects of suffering. A number of other measures with promising psychometric properties are now available to measure the particular dimensions of spiritual suffering.

\section{ACKNOWLEDGMENTS}

This project received funding from the Australian Government through Cancer Australia.

\section{REFERENCES}

Abbey, J.G., Rosenfeld, B., Pessin, H., et al. (2006). Hopelessness at the end of life: The utility of the hopelessness scale with terminally ill cancer patients. British Journal of Health Psychology, 11, 173-183.

Abernethy, A.P., Herndon II, J.E., Coan, A., et al. (2010). Phase 2 pilot study of pathfinders: A psychosocial intervention for cancer patients. Supportive Care in Cancer, $18,893-898$.

Adunsky, A., Aminoff, B.Z., Arad, M., et al. (2008). MiniSuffering State Examination: Suffering and survival of end-of-life cancer patients in a hospice setting. The American Journal of Hospice \& Palliative Medicine, 24, 493-498.

Aminoff, B.Z., Purits, E., Noy, S., et al. (2004). Measuring the suffering of end-stage dementia: Reliability and validity of the Mini-Suffering State Examination. Archives of Gerontology and Geriatrics, 38(2), 123-130.

Ando, M., Morita, T., Akechi, T., et al. (2010). Efficacy of short-term life-review interviews on the spiritual wellbeing of terminally ill cancer patients. Journal of Pain and Symptom Management, 39, 993-1002.

Antoni, M.H., Lehman, J.M., Kilbourn, K.M., et al. (2001). Cognitive-behavioral stress management intervention decreases the prevalence of depression and enhances benefit finding among women under treatment for early-stage breast cancer. Health Psychology, 20(1), 20.

Antonovsky, A. (1993). The structure and properties of the sense of coherence scale. Social Science \& Medicine, 36(6), 725-733.

Arman, M., Rehnsfeldt, A., Lindholm, L., et al. (2004). Suffering related to health care: A study of breast cancer patients' experiences. International Journal of Nursing Practice, 10, 248-256.

Axelsson, B. \& Sjoden, P.O. (1999). Assessment of quality of life in palliative care: Psychometric properties of a short questionnaire. Acta Oncologica, 38, 229-237.

Barton-Burke, M., Barreto Jr., R.C. \& Archibald, L.I.S. (2008). Suffering as a multicultural cancer experience. Seminars in Oncology Nursing, 24, 229-236.

Bayes, R., Limonero, J., Barreto, P., et al. (1996). A way to screen for suffering in palliative care. Journal of Palliative Care, 13(2), 22-26.

Beck, A.T., Weissman, A., Lester, D., et al. (1974). The measurement of pessimism: The hopelessness scale. Journal of Consulting and Clinical Psychology, 42(6), 861.

Beck, A.T., Steer, R.A., Beck, J.S., et al. (1993). Hopelessness, depression, suicidal ideation, and clinical diagnosis of depression. Suicide and Life-Threatening Behavior, 23(2), 139-145.

Best, M., Aldridge, L., Butow, P., et al. (2014). Conceptualization of suffering in cancer: A systematic literature review. In press.

Blinderman, C.D. \& Cherny, N.I. (2005). Existential issues do not necessarily result in existential suffering: Lessons from cancer patients in Israel. Palliative Medicine, 19(5), 371-380.

Boston, P., Bruce, A. \& Schreiber, R. (2011). Existential suffering in the palliative care setting: An integrated literature review. Journal of Pain and Symptom Management, 41(3), 604-618.

Bower, J.E., Meyerowitz, B.E., Desmond, K.A., et al. (2005). Perceptions of positive meaning and vulnerability following breast cancer: Predictors and outcomes among long-term breast cancer survivors. Annals of Behavioral Medicine, 29, 236-245.

Breitbart, W., Rosenfeld, B., Gibson, C., et al. (2010). Meaning-centered group psychotherapy for patients with advanced cancer: A pilot randomized controlled trial. Psycho-Oncology, 19, 21-28.

Breitbart, W., Poppito, S., Rosenfeld, B., et al. (2012). Pilot randomized controlled trial of individual meaning-centered psychotherapy for patients with advanced cancer. Journal of Clinical Oncology, 30(12), 1304-1309.

Bresnahan, J.F. \& Merrill, J.M. (2000). Religious/spiritual concerns in caring for the cancer patient. Cancer Treatment and Research, 102, 39-47.

Büchi, S. \& Sensky, T. (1999). PRISM: Pictorial Representation of Illness and Self Measure. A brief nonverbal measure of illness impact and therapeutic aid in psychosomatic medicine. Psychosomatics, 40, 314-320.

Büchi, S., Sensky, T., Sharpe, L. \& Timberlake, N. (1998). Graphic representation of illness: A novel method of measuring patients' perceptions of the impact of illness. Psychotherapy and Psychosomatics, 67(4-5), 222-225.

Büchi, S., Buddeberg, C., Klaghofer, R., et al. (2002). Preliminary validation of PRISM (Pictorial Representation of Illness and Self Measure): A brief method to assess suffering. Psychotherapy and Psychosomatics, 71(6), 333-341.

Byock, I. \& Merriman, M. (1998). Measuring quality of life for patients with terminal illness: The Missoula-VITAS quality of life index. Palliative Medicine, 12(4), 231-244. 
Canada, A.L., Murphy, P.E., Fitchett, G., et al. (2008). A three-factor model for the FACIT-Sp. Psycho-Oncology, 17(9), 908-916.

Carlson, L.E. \& Bultz, B.D. (2003). Cancer distress screening: Needs, models, and methods. Journal of Psychosomatic Research, 55(5), 403-409.

Carlson, L.E., Waller, A. \& Mitchell, A.J. (2012). Screening for distress and unmet needs in patients with cancer: Review and recommendations. Journal of Clinical Oncology, 30(11), 1160-1177.

Cassell, E.J. (1982). The nature of suffering and the goals of medicine. The New England Journal of Medicine, 306(11), 639-645.

Chan, T.H., Ho, R.T. \& Chan, C.L. (2007). Developing an outcome measurement for meaning-making intervention with Chinese cancer patients. Psycho-Oncology, 16, 843-850.

Chandwani, K.D., Thornton, B., Perkins, G.H., et al. (2010). Yoga improves quality of life and benefit finding in women undergoing radiotherapy for breast cancer. Journal of the Society for Integrative Oncology, 8, 43-55.

Chen, M.L. (1999). Validation of the structure of the perceived meanings of cancer pain inventory. Journal of Advanced Nursing, 30, 344-351.

Cherny, N.I., Coyle, N. \& Foley, K.M. (1994). Suffering in the advanced cancer patient: A definition and taxonomy. Journal of Palliative Care, 10(2), 57-70.

Chio, C.-C., Shih, F.-J., Chiou, J.-F., et al. (2008). The lived experiences of spiritual suffering and the healing process among Taiwanese patients with terminal cancer. Journal of Clinical Nursing, 17, 735-743.

Cockram, C.A., Doros, G. \& de Figueiredo, J.M. (2009). Diagnosis and measurement of subjective incompetence: The clinical hallmark of demoralization. Psychotherapy and Psychosomatics, 78, 342-345.

Cohen, S.R. \& Mount, B.M. (2000). Living with cancer: "Good" days and "bad" days. What produces them? Can the McGill Quality of Life Questionnaire distinguish between them? Cancer, 89, 1854-1865.

Cohen, S.R., Mount, B.M., Tomas, J.J., et al. (1996). Existential well-being is an important determinant of quality of life: Evidence from the McGill Quality of Life Questionnaire. Cancer, 77, 576-586.

Cohen, S.R., Mount, B.M., Bruera, E., et al. (1997). Validity of the McGill Quality of Life Questionnaire in the palliative care setting: A multi-centre Canadian study demonstrating the importance of the existential domain. Palliative Medicine, 11, 3-20.

Crumbaugh, J.C. \& Maholick, L.T. (1964). An experimental study in existentialism: The psychometric approach to Frankl's concept of noogenic neurosis. Journal of Clinical Psychology, 20(2), 200-207.

Delbar, V. \& Benor Dan, E. (2001). Impact of nursing intervention on cancer patients' ability to cope. Journal of Psychosocial Oncology, 19, 57-75.

den Oudsten, B.L., van Heck, G.L., van der Steeg, A.F., et al. (2009). The WHOQoL-100 has good psychometric properties in breast cancer patients. Journal of Clinical Epidemiology, 62, 195-205.

Ellison, C. (1983). Spiritual well-being: Conceptualization and measurement. Journal of Psychology and Theology, 11(4), 330-340.

Evers, A.W., Kraaimaat, F.W., van Lankveld, W., et al. (2001). Beyond unfavorable thinking: The illness cognition questionnaire for chronic diseases. Journal of Consulting and Clinical Psychology, 69(6), 1026.

Fallah, R., Golzari, M., Dastani, M., et al. (2011). Integrating spirituality into a group psychotherapy program for women surviving from breast cancer. Iranian Journal of Cancer Prevention, 4, 142-148.

Fegg, M.J., Kramer, M., l'Hoste, S., et al. (2008). The Schedule for Meaning in Life Evaluation (SMiLE): Validation of a new instrument for meaning-in-life research. Journal of Pain and Symptom Management, 35(4), 356-364.

Ferrans, C.E. (1990). Development of a quality of life index for patients with cancerOncology Nursing Forum, 17(3 Suppl.), 15-21.

Ferrans, C.E. \& Powers, M. J. (1985). Quality of life index: Development and psychometric properties. Advances in Nursing Science, 8(1), 15-24.

Ferrans, C.E. \& Powers, M.J. (1992). Psychometric assessment of the quality of life index. Research in Nursing \& Health, 15(1), 29-38.

Ferrell, B.R. (1993). To know suffering. Oncology Nursing Forum, 20, 1471-1477.

Ferrell, B.R., Dow, K.H. \& Grant, M. (1995). Measurement of the quality of life in cancer survivors. Quality of Life Research, 4, 523-531.

Fife, B.L. (1995). The measurement of meaning in illness. Social Science \& Medicine, 40, 1021-1028.

Fitzpatrick, R., Davey, C., Buxton, M., et al. (1998). Evaluating patient-based outcome measures for use in clinical trials. Health Technology Assessment, 2(14), 1-76.

Fortner, B., Okon, T., Schwartzberg, L., et al. (2003). The Cancer Care Monitor: Psychometric content evaluation and pilot testing of a computer-administered system for symptom screening and quality of life in adult cancer patients. Journal of Pain and Symptom Management, 26(6), 1077-1092.

Henderson, V.P., Clemow, L., Massion, A.O., et al. (2012). The effects of mindfulness-based stress reduction on psychosocial outcomes and quality of life in early-stage breast cancer patients: A randomized trial. Breast Cancer Research and Treatment, 131, 99-109.

Henoch, I. \& Danielson, E. (2009). Existential concerns among patients with cancer and interventions to meet them: An integrative literature review. Psycho-Oncolo$g y, 18,225-236$.

Henoch, I., Axelsson, B. \& Bergman, B. (2010). The Assessment of Quality of Life at the End of Life (AQEL) questionnaire: A brief but comprehensive instrument for use in patients with cancer in palliative care. Quality of Life Research, 19, 739-750.

Henry, M., Cohen, S.R., Lee, V., et al. (2010). The MeaningMaking intervention (MMi) appears to increase meaning in life in advanced ovarian cancer: A randomized controlled pilot study. Psycho-Oncology, 19, 1340-1347.

Herth, K. (1991). Development and refinement of an instrument to measure hope. Scholarly Inquiry for Nursing Practice, 5(1), 39-51.

Herth, K. (1992). Abbreviated instrument to measure hope: Development and psychometric evaluation. Journal of Advanced Nursing, 17(10), 1251-1259.

Herth, K. (2000). Enhancing hope in people with a first recurrence of cancer. Journal of Advanced Nursing, 1431-1441.

Highfield, M.F. (1992). Spiritual health of oncology patients: Nurse and patient perspectives. Cancer Nursing, 15(1), 1-8.

Hsiao, F.H., Jow, G.M., Kuo, W.H., et al. (2012). The effects of psychotherapy on psychological well-being and diurnal cortisol patterns in breast cancer survivors. Psychotherapy and Psychosomatics, 81, 173-182.

Hungelmann, J., Kenkel-Rossi, E., Klassen, L., et al. (1996). Focus on spiritual well-being: Harmonious 
interconnectedness of mind-body-spirit. Use of the JAREL Spiritual Well-Being Scale: Assessment of spiritual well-being is essential to the health of individuals. Geriatric Nursing, 17(6), 262-266.

Jaarsma T., A., Pool, G., Ranchor Adelita, V., et al. (2007). The concept and measurement of meaning in life in Dutch cancer patients. Psycho-Oncology, 16, 241-248.

Jacobsen, J.C., Vanderwerker, L.C., Block, S.D., et al. (2006). Depression and demoralization as distinct syndromes: Preliminary data from a cohort of advanced cancer patients. Indian Journal of Palliative Care, 12(1), 8-15.

Janoff-Bulman, R. (1989). Assumptive worlds and the stress of traumatic events: Applications of the schema construct. Social Cognition, 7(2), 113-136.

Jim, H.S., Purnell, J.Q., Richardson, S.A., et al. (2006) Measuring meaning in life following cancer. Quality of Life Research, 15, 1355-1371.

Johnson, M.E., Piderman, K.M., Sloan, J.A., et al. (2007). Measuring spiritual quality of life in patients with cancer. The Journal of Supportive Oncology, 5, 437-442.

Kahn, D.L. \& Steeves, R.H. (1995). The significance of suffering in cancer care. Seminars in Oncology Nursing, 11, $9-16$.

Kelly, B., McClement, S. \& Chochinov, H.M. (2006). Measurement of psychological distress in palliative care. Palliative Medicine, 20(8), 779-789.

Kissane, D.W., Wein, S., Love, A., et al. (2004). The Demoralization Scale: A report of its development and preliminary validation. Journal of Palliative Care, 20, 269-276.

Krikorian, A., Limonero, J.T. \& Corey, M.T. (2013). Suffering assessment: A review of available instruments for use in palliative care. Journal of Palliative Medicine, 16(2), 130-142.

Kroz, M., Bussing, A., von Laue, H.B., et al. (2009). Reliability and validity of a new scale on internal coherence (ICS) of cancer patients. Health and Quality of Life Outcomes, 7, 59 .

Ku, Y.-L., Kuo, S.-M. \& Yao, C.-Y. (2010). Establishing the validity of a spiritual distress scale for cancer patients hospitalized in southern Taiwan. International Journal of Palliative Nursing, 16, 134-138.

Lehmann, V., Oerlemans, S., van de Poll-Franse, L.V., et al. (2011). Suffering in long-term cancer survivors: An evaluation of the PRISM-R2 in a population-based cohort. Quality of Life Research, 20, 1645-1654.

Leung, K.-K., Chiu, T.-Y. \& Chen, C.-Y. (2006). The influence of awareness of terminal condition on spiritual well-being in terminal cancer patients. Journal of Pain and Symptom Management, 31, 449-456.

Lo, C., Burman, D., Swami, N., et al. (2011). Validation of the QUAL-EC for assessing quality of life in patients with advanced cancer. European Journal of Cancer, $47,554-560$.

Locke, D.E., Decker, P.A., Sloan, J.A., et al. (2007). Validation of single-item linear analog scale assessment of quality of life in neuro-oncology patients. Journal of Pain and Symptom Management, 34, 628-638.

Mack, J.W., Nilsson, M., Balboni, T., et al. (2008). Peace, equanimity, and acceptance in the cancer experience (PEACE): Validation of a scale to assess acceptance and struggle with terminal illness. Cancer, 112, 2509-2517.

Mayers, A.M., Khoo, S.T. \& Svartberg, M. (2002). The Existential Loneliness Questionnaire: Background, development, and preliminary findings. Journal of Clinical Psychology, 58(9), 1183-1193.
McMillan, S.C. \& Weitzner, M. (1998). Quality of life in cancer patients: Use of a revised Hospice Index. Cancer Practice, 6, 282-288.

Melton, A.M. \& Schulenberg, S.E. (2008). On the measurement of meaning: Logotherapy's empirical contributions to humanistic psychology. The Humanistic Psychologist, 36(1), 31-44.

Miller, J.F. \& Powers, M.J. (1988). Development of an instrument to measure hope. Nursing Research, 37(1), $6-10$.

Moore, R.J., Chamberlain, R.M. \& Khuri, F.R. (2004). Communicating suffering in primary stage head and neck cancer. European Journal of Cancer Care, 13, 53-64.

Mullane, M., Dooley, B., Tiernan, E. \& Bates, U. (2009). Validation of the Demoralization Scale in an Irish advanced cancer sample. Palliative \& Supportive Care, 7, $323-330$

Murphy, P.E., Canada, A.L., Fitchett, G., et al. (2010). An examination of the 3-factor model and structural invariance across racial/ethnic groups for the FACIT-Sp: A report from the American Cancer Society's Study of Cancer Survivors-II (SCS-II). Psycho-Oncology, 19(3), 264-272.

Nekolaichuk, C.L. \& Bruera, E. (2004). Assessing hope at the end of life: Validation of an experience of hope scale in advanced cancer patients. Palliative \& Supportive Care, 2, 243-253.

Nekolaichuk, C.L., Jevne, R.F. \& Maguire, T.O. (1999). Structuring the meaning of hope in health and illness. Social Science \& Medicine, 48(5), 591-605.

Nissim, R., Flora, D.B., Cribbie, R.A., et al. (2010). Factor structure of the Beck Hopelessness Scale in individuals with advanced cancer. Psycho-Oncology, 19, 255-263.

Northouse, L.L., Mood, D.W., Schafenacker, A., et al. (2007). Randomized clinical trial of a family intervention for prostate cancer patients and their spouses. Cancer, 110, 2809-2818.

Nowotny, M. (1989). Assessment of hope in patients with cancer: Development of an instrument. Oncology Nursing Forum, 16(1), 57-61.

Pang, S.M.C., Chan, K.-S., Chung, B.P.M., et al. (2005). Assessing quality of life of patients with advanced chronic obstructive pulmonary disease in the end of life. Journal of Palliative Care, 21, 180-187.

Passik, S.D., Inman, A., Kirsh, K., et al. (2003). Initial validation of a scale to measure purposelessness, understimulation, and boredom in cancer patients: Toward a redefinition of depression in advanced disease. Palliative \& Supportive Care, 1, 41-50.

Penedo, F.J., Molton, I., Dahn, J.R., et al. (2006). A randomized clinical trial of group-based cognitive-behavioral stress management in localized prostate cancer: Development of stress management skills improves quality of life and benefit finding. Annals of Behavioral Medicine, 31(3), 261-270.

Peterman, A.H., Min, G.F.D., Brady, M.J., et al. (2002). Measuring spiritual well-being in people with cancer: The functional assessment of chronic illness therapy. Spiritual Well-Being Scale (FACIT-Sp). Annals of Behavioral Medicine, 24(1), 49-58.

Phillips-Salimi, C.R., Haase, J.E., Kintner, E.K., et al. (2007). Psychometric properties of the Herth Hope Index in adolescents and young adults with cancer. Journal of Nursing Measurement, 15(1), 3-23.

Reed, P.G. (1987). Spirituality and well-being in terminally ill hospitalized adults. Research in Nursing \& Health, 10(5), 335-344. 
Reed, P.G. (1991). Self-transcendence and mental health in oldest-old adults. Nursing Research, 40(1), 5-11.

Reker, G. (1992). Manual of the Life Attitude Profile-Revised (LAP-R). Peterborough, Ontario: Student Psychologists Press.

Reker, G. (1996). Manual of the Sources of Meaning Profile-Revised (SOMP-R). Trent, Ontario: Department of Psychology, Trent University.

Reker, G.T. \& Peacock, E.J. (1981). The Life Attitude Profile (LAP): A multidimensional instrument for assessing attitudes toward life. Canadian Journal of Behavioral Science, 13(3), 264.

Rodgers, B.L. \& Cowles, K.V. (1997). A conceptual foundation for human suffering in nursing care and research. Journal of Advanced Nursing, 25(5), 1048-1053.

Rodin, G.M. (2003). Suffering and adaptation to cancer: What to measure and when to intervene. Journal of Psychosomatic Research, 55(5), 399-401.

Rosenfeld, B., Breitbart, W., Stein, K., et al. (1999). Measuring desire for death among patients with HIV/AIDS: The Schedule of Attitudes toward Hastened Death. The American Journal of Psychiatry, 156(1), 94-100.

Rosenfeld, B., Breitbart, W., Galietta, M., et al. (2000). The schedule of attitudes toward hastened death: Measuring desire for death in terminally ill cancer patients. Cancer, 88, 2868-2875.

Rosenfeld, B., Pessin, H., Lewis, C., et al. (2011). Assessing hopelessness in terminally ill cancer patients: Development of the Hopelessness Assessment in Illness Questionnaire. Psychological Assessment, 23, 325-336.

Rustoen, T., Wiklund, I., Hanestad, B.R., et al. (1998). Nursing intervention to increase hope and quality of life in newly diagnosed cancer patients. Cancer Nursing, 21, 235-245.

Salmon, P., Manzi, F. \& Valori, R.M. (1996). Measuring the meaning of life for patients with incurable cancer: The life evaluation questionnaire (LEQ). European Journal of Cancer, 32A, 755-760.

Selman, L., Siegert, R.J., Higginson, I.J., et al. (2011). The MVQoLI successfully captured quality of life in African palliative care: A factor analysis. Journal of Clinical Epidemiology, 64, 913-924.

Selman, L., Siegert, R.J., Higginson, I.J., et al. (2012). The "Spirit 8" successfully captured spiritual well-being in African palliative care: Factor and Rasch analysis. Journal of Clinical Epidemiology, 65(4), 434-443.

Sherman, D.W., Xiang, Y.Y., McSherry, C., et al. (2005). Spiritual well-being as a dimension of quality of life for patients with advanced cancer and AIDS and their family caregivers: Results of a longitudinal study. The American Journal of Hospice \& Palliative Medicine, 22, 349-362.

Snyder, C.R., Harris, C., Anderson, J.R., et al. (1991). The will and the ways: Development and validation of an individual-differences measure of hope. Journal of Personality and Social Psychology, 60(4), 570.
Starck, P.L. (1983). Patients' perceptions of the meaning of suffering. International Forum for Logotherapy, 6(2), 110-116.

Steger, M.F., Frazier, P., Oishi, S., et al. (2006). The meaning in life questionnaire: Assessing the presence of and search for meaning in life. Journal of Counseling Psychology, 53(1), 80.

Steinhauser, K., Voils, C., Clipp, E., et al. (2006). "Are you at peace?": One item to probe spiritual concerns at the end of life. Archives of Internal Medicine, 166(1), 101-105.

Strang, P. (1997). Existential consequences of unrelieved cancer pain. Palliative Medicine, 11, 299-305.

Sulmasy, D.P. (2006). Spiritual issues in the care of dying patients: “. . It's okay between me and God." The Journal of the American Medical Association, 296(11), 1385-1392.

Thomas, J.C., Burton, M., Griffin, M.T.Q., et al. (2010) Self-transcendence, spiritual well-being, and spiritual practices of women with breast cancer. Journal of Holistic Nursing, 28, 115-122.

Tomich, P.L. \& Helgeson, V.S. (2002). Five years later: A cross-sectional comparison of breast cancer survivors with healthy women. Psycho-Oncology, 11, 154-169.

van Wegberg, B., Bacchi, M., Heusser, P., et al. (1998). The cognitive-spiritual dimension: An important addition to the assessment of quality of life. Validation of a questionnaire (SELT-M) in patients with advanced cancer. Annals of Oncology, 9, 1091-1096.

Wein, S. (2011). Impact of culture on the expression of pain and suffering. Journal of Pediatric Hematology/Oncology, 33 (Suppl. 2), S105-S107.

WHOQoL SRPB Group (2006). A cross-cultural study of spirituality, religion, and personal beliefs as components of quality of life. Social Science \& Medicine, 62, 1486-1497.

Williams, B.R. (2004). Dying young, dying poor: A sociological examination of existential suffering among low-socioeconomic status patients. Journal of Palliative Medicine, 7(1), 27-37.

Wilson, K.G., Graham, I.D., Viola, R.A., et al. (2004). Structured interview assessment of symptoms and concerns in palliative care. Canadian Journal of Psychiatry, 49, $350-358$

Wong, P.T. (1998). Implicit theories of meaningful life and the development of the personal meaning profile. Mahwah, NJ: Lawrence Erlbaum Associates.

Wouters, E.J.M., Reimus, J.L.M., van Nunen, A.M.A., et al. (2008). Suffering quantified? Feasibility and psychometric characteristics of two revised versions of the Pictorial Representation of Illness and Self Measure (PRISM). Behavioral Medicine, 34, 65-78.

Wyatt, G., Kurtz, M.E., Friedman, L.L., et al. (1996). Preliminary testing of the Long-Term Quality of Life (LTQL) instrument for female cancer survivors. Journal of Nursing Measurement, 4, 153-170.

Younger, J.B. (1995). The alienation of the sufferer. Advances in Nursing Science, 17(4), 53-72. 